UMD-PP-05-023

OSU-HEP-04-10

\title{
Tying in CP and Flavor Violations with Fermion Masses and Neutrino Oscillations
}

\author{
K.S. Babu ${ }^{\mathrm{a}}$, Jogesh C. Pati ${ }^{\mathrm{b}}$, Parul Rastogi ${ }^{\mathrm{b}}$ \\ ${ }^{a}$ Oklahoma Center for High Energy Physics, Department of Physics \\ Oklahoma State University, Stillwater, OK 74078, USA \\ ${ }^{b}$ Department of Physics, University of Maryland, College Park MD 20742, USA \\ October 13, 2004.
}

\begin{abstract}
In this paper we explore the possibility that (a) fermion masses, (b) neutrino oscillations, (c) CP non-conservation and (d) flavor violations get intimately linked to each other within supersymmetric grand unification based on $\mathrm{SO}(10)$ or an effective $\mathrm{G}(224)=S U(2)_{L} \times S U(2)_{R} \times S U(4)^{c}$ symmetry. We extend the framework proposed previously by Babu, Pati and Wilczek (BPW) which successfully described fermion masses and neutrino oscillations, to include CP violation. Assuming flavor universal SUSY breaking parameters at $M^{*} \gtrsim M_{G U T}$, and that CP violation arises through phases in the fermion mass matrices, we show how the presence of GUT threshold induces new and calculable CP and flavor violations. Including SM and SUSY contributions, we find that the BPW framework can correctly account for the observed flavor and/or CP violations in $\Delta m_{K}, \Delta m_{B_{d}}, S\left(B_{d} \rightarrow J / \psi K_{S}\right)$ and $\epsilon_{K}$. While SUSY-contribution is small $\left(\lesssim\right.$ few\%) for the first three quantities, that to $\epsilon_{K}$ is sizable $(\sim 20-25 \%)$ and negative (as desired) compared to that of the SM. The model predicts $S\left(B_{d} \rightarrow \phi K_{S}\right)$ to be in the range $+(0.65-0.73)$, close to the SM prediction. The model yields $\operatorname{Re}\left(\epsilon^{\prime} / \epsilon\right)_{S U S Y} \approx+(4-14) \times 10^{-4}$; the relevance of this contribution can be assessed only when the associated matrix elements are known reliably. The model also predicts that the electric dipole moments of the neutron and the electron should be discovered with improvements in current limits by factors of 10 to 100 .
\end{abstract}




\section{Introduction}

Ongoing studies of $\mathrm{CP}$ and flavor violations may well turn out to provide some important clues to physics beyond the standard model (SM). Strong agreement with the expectations of the SM would provide severe constraints on new physics, while departures could suggest the nature and the threshold for such new physics. On the experimental side there are now four well measured quantities reflecting $\mathrm{CP}$ and/or $\Delta F=2$ flavor violations. They are: ${ }^{1}$

$$
\Delta m_{K}, \epsilon_{K}, \Delta m_{B_{d}} \text { and } S\left(B_{d} \rightarrow J / \Psi K_{S}\right)
$$

where $S\left(B_{d} \rightarrow J / \Psi K_{S}\right)$ denotes the asymmetry parameter in $\left(B_{d}\right.$ versus $\left.\overline{B_{d}}\right) \rightarrow J / \Psi K_{S}$ decays. It is indeed remarkable that the observed values including the signs of all four quantities as well as the empirical lower limit on $\Delta m_{B_{s}}$ can consistently be realized within the standard CKM model for a single choice of the Wolfenstein parameters [1]:

$$
\bar{\rho}_{W}=0.178 \pm 0.046 ; \bar{\eta}_{W}=0.341 \pm 0.028
$$

This fit is obtained using the observed values of $\epsilon_{K}=2.27 \times 10^{-3}, V_{u s}=0.2240 \pm 0.0036$, $\left|V_{u b}\right|=(3.30 \pm 0.24) \times 10^{-3},\left|V_{c b}\right|=(4.14 \pm 0.07) \times 10^{-2},\left|\Delta m_{B_{d}}\right|=(3.3 \pm 0.06) \times 10^{-13} \mathrm{GeV}$ and $\Delta m_{B_{d}} / \Delta m_{B_{s}}>0.035$, and allowing for uncertainties in the hadronic matrix elements of up to $15 \%$. One can then predict the asymmetry parameter $S\left(B_{d} \rightarrow J / \Psi K_{S}\right)$ in the $\mathrm{SM}$ to be $\approx 0.685 \pm 0.052[1,2]$. This agrees remarkably well with the observed value $S\left(B_{d} \rightarrow J / \Psi K_{S}\right)_{\text {expt. }}=0.734 \pm 0.054$, representing an average of the BABAR and BELLE results [3]. This agreement of the SM prediction with the data in turn poses a challenge for physics beyond the SM, especially for supersymmetric grand unified (SUSY GUT) models, as these generically possess new sources of CP and flavor violations beyond those of the SM.

The purpose of this paper is to address the issues of $\mathrm{CP}$ and flavor violations in conjunction with those of fermion masses and neutrino oscillations, in the context of SUSY grand unification. In particular, our goal would be to obtain a unified description, in accord with observations, of all four phenomena: (i) CP non-conservation, (ii) flavor violation, (iii) masses and mixings of quarks and leptons, as well as (iv) neutrino oscillations, within a single predictive framework based on SUSY grand unification.

\footnotetext{
${ }^{1} \epsilon_{K}^{\prime}$ reflecting direct $\Delta F=1 \mathrm{CP}$ violation is well measured, but its theoretical implications are at present unclear due to uncertainties in the matrix element. We discuss this later.
} 
In this regard, (a) the observed quantum numbers of the members of a family, (b) gauge coupling unification, (c) neutrino oscillations, as well as (d) the likely need for leptogenesis [4] as a prelude to baryogenesis, together, suggest that the SM gauge symmetry very likely emerges, near the GUT scale $M_{U} \sim 2 \times 10^{16} \mathrm{GeV}$, from the spontaneous breaking of a higher gauge symmetry that possesses the symmetry SU(4)-color [5]. The higher gauge symmetry in 4D could be either $\mathrm{SO}(10)$ [6], or an effective (presumably string derived) symmetry $\mathrm{G}(224)=S U(2)_{L} \times S U(2)_{R} \times S U(4)^{c}[5]$. The need for $\mathrm{SU}(4)$-color arises because it provides: (a) the right-handed neutrino $\left(\nu_{R}\right)$ as a compelling member of each family, (b) $B-L$ as a local symmetry, and (c) the Dirac mass of $\nu_{\tau}$ in terms of the top-quark mass. These three ingredients, together with the SUSY unification scale, seem to be rather crucial [7] to an understanding of the neutrino masses via the seesaw mechanism [8] and implementing baryogenesis via leptogenesis [4]. The theory thus described may be viewed to have emerged in $4 \mathrm{D}$ from a string/M theory near the string scale $M_{s t} \gtrsim M_{G U T}{ }^{2}$. It should of course possess weak scale supersymmmetry so as to avoid unnatural fine tuning in the Higgs mass and ensure gauge coupling unification.

A predictive framework based on the symmetry $\mathrm{SO}(10)$ or $\mathrm{G}(224)$, and a minimal Higgs system which accomplish all these has been proposed in Ref. [9], which we refer to as the BPW model. This model describes the masses and mixings of all fermions including neutrinos by making the simplifying assumption that the fermion mass matrices are real and thus CP-conserving. Notwithstanding this assumption, the framework is found to be remarkably successful. In particular, it makes seven predictions involving fermion masses, CKM elements and neutrino oscillations, all in good accord with observations, to within $10 \%$ (see discussion in Sec. 2).

Now in general one would of course expect the entries in the fermion mass matrices to have phases because the VEVs of the relevant Higgs fields, and/or the effective Yukawa couplings, can well be complex. These in turn can induce CP violation through the SM/CKM interactions as well as through SUSY interactions involving sfermion/gaugino loops.

The question arises: Can the BPW-framework of Ref. [9], based on the supersymmetric $\mathrm{SO}(10)$ or $\mathrm{G}(224)$-symmetry, be extended, by allowing for phases in the fermion mass

\footnotetext{
${ }^{2}$ The relative advantages of $\mathrm{SO}(10)$ versus $\mathrm{G}(224)$ as effective $4 \mathrm{D}$ symmetries involving the issues of coupling unification on the one hand and doublet-triplet splitting on the other hand are discussed in [7].
} 
matrices, so as to yield net CP and flavor-violations, arising through both SM and SUSY interactions, in accord with observations, while still preserving its successes as regards fermion masses and neutrino oscillations? This is the question we pursue in this paper.

As we will see, these four phenomena - (i) fermion masses, (ii) neutrino oscillations, (iii) CP non-conservation, and (iv) flavor violations - get intimately linked to each other within the SUSY SO(10)/G(224) framework. Satisfying simultaneously the observed features of all four phenomena within such a predictive framework turns out, however, to be a non-trivial challenge to meet. One main purpose of the present paper is to show that the answer to the question raised above is in the affirmative. We defer the discussion of our results and predictions in this regard to section 5 .

Since CP violation would have its origin, in our model, entirely in the fermion mass matrices, and since the BPW framework presented in Ref. [9] has proven to be so successful in describing fermion masses and neutrino oscillations, we first briefly recall the salient features of this prior work in the next section. In the following section we extend the same so as to include $\mathrm{CP}$ violation.

\section{Fermion Masses and Neutrino Oscillations in $\mathrm{G}(224) / \mathrm{SO}(10)$ : A Brief Review of Prior Work}

The $3 \times 3$ Dirac mass matrices for the four sectors $(u, d, l, \nu)$ proposed in Ref. [9] were motivated in part by the notion that flavor symmetries [10] are responsible for the hierarchy among the elements of these matrices (i.e., for " $33 " \gg " 23 " \gg " 22 " \gg " 12 " \gg " 11 "$, etc.), and in part by the group theory of $\mathrm{SO}(10) / \mathrm{G}(224)$, relevant to a minimal Higgs system. Up to 
minor variants [11], they are as follows:

$$
\begin{gathered}
M_{u}=\left[\begin{array}{ccc}
0 & \epsilon^{\prime} & 0 \\
-\epsilon^{\prime} & \zeta_{22}^{u} & \sigma+\epsilon \\
0 & \sigma-\epsilon & 1
\end{array}\right] \mathcal{M}_{u}^{0} ; \quad M_{d}=\left[\begin{array}{ccc}
0 & \eta^{\prime}+\epsilon^{\prime} & 0 \\
\eta^{\prime}-\epsilon^{\prime} & \zeta_{22}^{d} & \eta+\epsilon \\
0 & \eta-\epsilon & 1
\end{array}\right] \mathcal{M}_{d}^{0} \\
M_{\nu}^{D}=\left[\begin{array}{ccc}
0 & -3 \epsilon^{\prime} & 0 \\
3 \epsilon^{\prime} & \zeta_{22}^{u} & \sigma-3 \epsilon \\
0 & \sigma+3 \epsilon & 1
\end{array}\right] \mathcal{M}_{u}^{0} ; \quad M_{l}=\left[\begin{array}{ccc}
0 & \eta^{\prime}-3 \epsilon^{\prime} & 0 \\
\eta^{\prime}+3 \epsilon^{\prime} & \zeta_{22}^{d} & \eta-3 \epsilon \\
0 & \eta+3 \epsilon & 1
\end{array}\right] \mathcal{M}_{d}^{0}
\end{gathered}
$$

These matrices are defined in the gauge basis and are multiplied by $\bar{\Psi}_{L}$ on left and $\Psi_{R}$ on right. For instance, the row and column indices of $M_{u}$ are given by $\left(\bar{u}_{L}, \bar{c}_{L}, \bar{t}_{L}\right)$ and $\left(u_{R}, c_{R}, t_{R}\right)$ respectively. Note the group-theoretic up-down and quark-lepton correlations: the same $\sigma$ occurs in $M_{u}$ and $M_{\nu}^{D}$, and the same $\eta$ occurs in $M_{d}$ and $M_{l}$. It will become clear that the $\epsilon$ and $\epsilon^{\prime}$ entries are proportional to $B-L$ and are antisymmetric in the family space (as shown above). Thus, the same $\epsilon$ and $\epsilon^{\prime}$ occur in both $\left(M_{u}\right.$ and $\left.M_{d}\right)$ and also in $\left(M_{\nu}^{D}\right.$ and $M_{l}$ ), but $\epsilon \rightarrow-3 \epsilon$ and $\epsilon^{\prime} \rightarrow-3 \epsilon^{\prime}$ as $q \rightarrow l$. Such correlations result in enormous reduction of parameters and thus in increased predictiveness. Such a pattern for the mass-matrices can be obtained, using a minimal Higgs system $\mathbf{4 5} \mathbf{5}_{H}, \mathbf{1 6}_{H}, \overline{\mathbf{1 6}}_{H}$ and $\mathbf{1 0}_{H}$ and a singlet $S$ of SO(10), through effective couplings as follows [12] (see Ref. [9] and [7] for details):

$$
\begin{aligned}
\mathcal{L}_{\text {Yuk }} & =h_{33} \mathbf{1 6}_{3} \mathbf{1 6}_{3} \mathbf{1 0}_{H}+\left[h_{23} \mathbf{1 6}_{2} \mathbf{1 6}_{3} \mathbf{1 0}_{H}(S / M)\right. \\
& \left.+a_{23} \mathbf{1 6}_{2} \mathbf{1 6}_{3} \mathbf{1 0}_{H}\left(\mathbf{4 5}_{H} / M^{\prime}\right)(S / M)^{p}+g_{23} \mathbf{1 6}_{2} \mathbf{1 6}_{3} \mathbf{1 6}_{H}^{d}\left(\mathbf{1 6}_{H} / M^{\prime \prime}\right)(S / M)^{q}\right] \\
& +\left[h_{22} \mathbf{1 6}_{2} \mathbf{1 6}_{2} \mathbf{1 0}_{H}(S / M)^{2}+g_{22} \mathbf{1 6}_{2} \mathbf{1 6}_{2} \mathbf{1 6}_{H}^{d}\left(\mathbf{1 6}_{H} / M^{\prime \prime}\right)(S / M)^{q+1}\right] \\
& +\left[g_{12} \mathbf{1 6}_{1} \mathbf{1 6}_{2} \mathbf{1 6}_{H}^{d}\left(\mathbf{1 6}_{H} / M^{\prime \prime}\right)(S / M)^{q+2}+a_{12} \mathbf{1 6}_{1} \mathbf{1 6}_{2} \mathbf{1 0}_{H}\left(\mathbf{4 5}_{H} / M^{\prime}\right)(S / M)^{p+2}\right]
\end{aligned}
$$

Typically we expect $M^{\prime}, M^{\prime \prime}$ and $M$ to be of order $M_{\text {string }}$ or (possibly) of order $M_{G U T}$ [13]. The VEV's of $\left\langle\mathbf{4 5}_{H}\right\rangle$ (along $\left.B-L\right),\left\langle\mathbf{1 6}_{H}\right\rangle=\left\langle\overline{\mathbf{1 6}}_{H}\right\rangle$ (along standard model singlet sneutrinolike component) and of the $\mathrm{SO}(10)$-singlet $\langle S\rangle$ are of the GUT-scale, while those of $\mathbf{1 0}_{H}$ and of the down type $\mathrm{SU}(2)_{L}$-doublet component in $\mathbf{1 6}_{H}$ (denoted by $\mathbf{1 6}_{H}^{d}$ ) are of the electroweak scale $[9,14]$. Depending upon whether $M^{\prime}\left(M^{\prime \prime}\right) \sim M_{\mathrm{GUT}}$ or $M_{\text {string }}$ (see [13]), the exponent $p(q)$ is either one or zero [15].

The entries 1 and $\sigma$ arise respectively from $h_{33}$ and $h_{23}$ couplings, while $\hat{\eta} \equiv \eta-\sigma$ and $\eta^{\prime}$ 
arise respectively from $g_{23}$ and $g_{12}$-couplings. The $(B-L)$-dependent antisymmetric entries $\epsilon$ and $\epsilon^{\prime}$ arise respectively from the $a_{23}$ and $a_{12}$ couplings. [Effectively, with $\left\langle\mathbf{4 5} \mathbf{5}_{H}\right\rangle \propto B-L$, the product $\mathbf{1 0} \mathbf{H}_{H} \times \mathbf{4} \mathbf{5}_{H}$ contributes as a $\mathbf{1 2 0}$, whose coupling is family-antisymmetric.] The small entry $\zeta_{22}^{u}$ arises from the $h_{22}$-coupling, while $\zeta_{22}^{d}$ arises from the joint contributions of $h_{22}$ and $g_{22}$-couplings. As discussed in [9], using some of the observed masses as inputs, one obtains $|\hat{\eta}| \sim|\sigma| \sim|\epsilon| \sim \mathcal{O}(1 / 10),\left|\eta^{\prime}\right| \approx$ few $\times 10^{-3}$ and $\left|\epsilon^{\prime}\right| \sim 2 \times 10^{-4}$. The success of the framework presented in Ref. [9] (which sets $\zeta_{22}^{u}=\zeta_{22}^{d}=0$ ) in describing fermion masses and mixings remains essentially unaltered if $\left|\left(\zeta_{22}^{u}, \zeta_{22}^{d}\right)\right| \leq(1 / 3)\left(10^{-2}\right)$ (say).

Such a hierarchical form of the mass-matrices, with $h_{33}$-term being dominant, is attributed in part to flavor gauge symmetry(ies) that distinguishes between the three families, and in part to higher dimensional operators involving for example $\left\langle\mathbf{4 5} \mathbf{5}_{H}\right\rangle / M^{\prime}$ or $\left\langle\mathbf{1 6}_{H}\right\rangle / M^{\prime \prime}$, which are suppressed by $M_{\mathrm{GUT}} / M_{\text {string }} \sim 1 / 10$, if $M^{\prime}$ and/or $M^{\prime \prime} \sim M_{\text {string. As an example, }}$. As a introduce just one $U(1)$-flavor gauge symmetry, together with a discrete $Z_{2}$-symmetry $D$, with one singlet $S$. The hierarchical form of the Yukawa couplings, in accord with Eqs. (3) and (4), would follow, for the case of $p=1, q=0$, if, for example, the $U(1)$ flavor charges are assigned as follows: ${ }^{3}$

$$
\begin{array}{cccccccc}
16_{3} & 16_{2} & \mathbf{1 6}_{1} & \mathbf{1 0}_{H} & \mathbf{1 6}_{H} & \overline{\mathbf{1 6}}_{H} & \mathbf{4 5 _ { H }} & \mathbf{S} \\
a & a+1 & a+2 & -2 a & -a-1 / 2 & -a & 0 & -1
\end{array} .
$$

All the fields are assumed to be even under the discrete symmetry $D$, except for $\mathbf{1 6}_{H}$ and $\overline{\mathbf{1 6}}_{H}$ which are odd. It is assumed that other fields are present that would make the $U(1)$ symmetry anomaly-free. With this assignment of charges, one would expect $\left|\zeta_{22}^{u, d}\right| \sim(\langle S\rangle / M)^{2}$; one may thus take $\left|\zeta_{22}^{u, d}\right| \sim(1 / 3) \times 10^{-2}$ without upsetting the success of Ref. [9]. In the same spirit, one would expect $\left|\zeta_{13}, \zeta_{31}\right| \sim(\langle S\rangle / M)^{2} \sim 10^{-2}$ and $\left|\zeta_{11}\right| \sim(\langle S\rangle / M)^{4} \sim 10^{-4}$ (say). where $\zeta_{11}, \zeta_{13}$, and $\zeta_{31}$ denote the "11", "13", and "31", elements respectively. The value of $a$ can get fixed by the presence of other operators (see Ref. [16]).

The Majorana masses of the RH neutrinos arise from effective couplings of the form $\mathcal{L}_{\mathrm{Maj}}=f_{i j} \mathbf{1 6}_{i} \mathbf{1 6}_{j} \overline{\mathbf{1 6}}_{H} \overline{\mathbf{1 6}}_{H} / M$, where the $f_{i j}$ 's include appropriate powers of $\langle S / M\rangle$ in accord

\footnotetext{
${ }^{3}$ An alternative assignment with $p=1, q=1$ would arise by choosing the charges: $(0,1,2,0,0,0,0$ and -1$)$ for $\left(\mathbf{1 6}_{3}, \mathbf{1 6}_{2}, \mathbf{1 6}_{1}, \mathbf{1 0}_{H}, \mathbf{1 6}_{H}, \overline{\mathbf{1 6}}_{H}, \mathbf{4 5}_{H}\right.$ and $S$ ) respectively. Another variant would be to assign a flavor charge of 3 (or a +3 in case of Eq. (5) to the first family $\mathbf{1 6}$, leaving all other charges as above. This would suppress "13", "31", "12", "21", and " $11 "$ entries by an order of magnitude relative to the other cases.
} 
with flavor-charge assignments of $\mathbf{1 6}_{i}$ and $\overline{\mathbf{1 6}}_{\mathbf{H}}$, and $M$ is expected to be of order string or reduced Planck-scale. From the flavor-charge assignments given in Eq. (5), it is clear that the "33" entry is leading and the other entries in the Majorana mass matrix have a relative hierarchy which is identical to that in the Dirac mass matrices. We refer the reader to [9] and [7] for a more detailed discussion of the neutrino sector. Here, we list only the results.

Ignoring possible phases in the parameters and thus the source of $\mathrm{CP}$ violation for a moment, and also setting $\zeta_{22}^{d}=\zeta_{22}^{u}=0$, as was done in Ref. [9], the parameters $\left(\sigma, \eta, \epsilon, \epsilon^{\prime}, \eta^{\prime}, \mathcal{M}_{u}^{0}\right.$, and $\left.\mathcal{M}_{d}^{0}\right)$ can be determined by using, for example, $m_{t}^{\text {phys }}=174 \mathrm{GeV}$, $m_{c}\left(m_{c}\right)=1.37 \mathrm{GeV}, m_{s}(1 \mathrm{GeV})=110-116 \mathrm{MeV}, m_{u}(1 \mathrm{GeV})=6 \mathrm{MeV}$, and the observed masses of $e, \mu$, and $\tau$ as inputs. One is thus led, for this CP conserving case, to the following fit for the parameters, and the associated predictions [9]:

$$
\begin{aligned}
\sigma & \approx 0.110, \quad \eta \approx 0.151, \quad \epsilon \approx-0.095, \quad\left|\eta^{\prime}\right| \approx 4.4 \times 10^{-3} \\
\epsilon^{\prime} & \approx 2 \times 10^{-4}, \quad \mathcal{M}_{u}^{0} \approx m_{t}\left(M_{X}\right) \approx 100 \mathrm{GeV}, \quad \mathcal{M}_{d}^{0} \approx m_{\tau}\left(M_{X}\right) \approx 1.1 \mathrm{GeV} .
\end{aligned}
$$

These output parameters remain stable to within $10 \%$ corresponding to small variations $(\lesssim 10 \%)$ in the input parameters of $m_{t}, m_{c}, m_{s}$, and $m_{u}$. These in turn lead to the following predictions for the quarks and light neutrinos [9], [7]:

$$
\begin{aligned}
& m_{b}\left(m_{b}\right) \approx 4.9 \mathrm{GeV}, \quad \sqrt{\Delta m_{23}^{2}} \approx m\left(\nu_{3}\right) \approx(1 / 24 \mathrm{eV})(1 / 2-2), \quad V_{c b} \approx 0.044, \\
& \sin ^{2} 2 \theta_{\nu_{\mu} \nu_{\tau}}^{\text {osc }} \approx 0.98-0.995,\left(\text { for } m\left(\nu_{2}\right) / m\left(\nu_{3}\right) \approx 1 / 10-1 / 5\right), \\
& \left|V_{u s}\right| \approx 0.20, \quad\left|\frac{V_{u b}}{V_{c b}}\right| \approx 0.07, \quad m_{d}(1 \mathrm{GeV}) \approx 8 \mathrm{MeV}
\end{aligned}
$$

It has been noted $[7,16]$ that small non-seesaw contribution to $\nu_{L}^{e} \nu_{L}^{\mu}$ mass term $(\sim$ few $\times 10^{-3} \mathrm{eV}$ ) which can arise through higher dimensional operators, but which have been ignored in the analysis given above, can lead quite plausibly to large $\nu_{e}-\nu_{\mu}$ oscillation angle in accord with the LMA MSW solution for the solar neutrino problem. Leaving aside therefore the question of the $\nu_{e}-\nu_{\mu}$ oscillation angle, it seems quite remarkable that all seven predictions in Eq. (77) agree with observations to within 10\%. Particularly intriguing is the $(B-L)$-dependent group-theoretic correlation between $V_{c b}$ and $\theta_{\nu_{\mu} \nu_{\tau}}^{o s c}$, which explains simultaneously why one is small $\left(V_{c b}\right)$ and the other is so large $\left(\theta_{\nu_{\mu \nu} \nu_{\tau}}^{o s c}\right)[7,9]$. That in turn provides some confidence in the gross pattern of the Dirac mass matrices presented above and motivates the study of $\mathrm{CP}$ and flavor violations within the same framework. This is what we do in the next section. 


\section{Phases in the Fermion Mass Matrices: The Origin of CP viola- tion}

In the work of Ref. [9] reviewed above, the parameters $\left(\sigma, \epsilon, \eta, \epsilon^{\prime}, \eta^{\prime}\right.$ etc.) entering into the fermion mass matrices were assumed to be real, for simplicity, and thereby (at least) the $\mathrm{SM}$ interactions were rendered CP-conserving ${ }^{4}$. Noting that the VEVs of the Higgs fields ${ }^{5}$ and/or the effective Yukawa couplings can well be complex, however, we now propose to extend the $\mathrm{SO}(10) / \mathrm{G}(224)$ framework reviewed above to include $\mathrm{CP}$ violation by allowing for these parameters to have phases.

Given the empirical constraints on (i) CP and flavor violations, as well as (ii) fermion masses and (iii) neutrino oscillations, on the one hand, and (iv) the group-theoretical constraints of the $\mathrm{SO}(10) / \mathrm{G}(224)$ framework on the other, it is of course not at all clear, a priori, whether any choice of phases and variations in the parameters of the fermion mass matrices presented above can yield observed $\mathrm{CP}$ and flavor-violations, and simultaneously preserve the successes of the framework of [9] as regards fermion masses and neutrino oscillations. That is the issue we now explore. We choose to diagonalize the quark mass matrices $M_{u}$ and $M_{d}$ at the GUT scale $\sim 2 \times 10^{16} \mathrm{GeV}$, by bi-unitary transformations - i.e.

$$
M_{d}^{\text {diag }}=X_{L}^{d \dagger} M_{d} X_{R}^{d} \text { and } M_{u}^{\text {diag }}=X_{L}^{u \dagger} M_{u} X_{R}^{u}
$$

with phases of $q_{L, R}^{i}$ chosen such that the eigenvalues are real and positive and that the CKM matrix $V_{C K M}$ (defined below) has the Wolfenstein form [17]). Utilizing the hierarchical nature of the mass matrices, one can obtain (approximate) analytic expressions for the diagonalizing matrices. They are:

$$
X_{L}^{d} \simeq\left[\begin{array}{ccc}
e^{-i\left(\phi_{\eta-\epsilon}\right)} & \left|\eta^{\prime} / X_{d}\right| e^{-i\left(\phi_{\eta-\epsilon}+\zeta_{u s}\right)} & \eta^{\prime}|\eta-\epsilon| e^{-i\left(\phi_{\eta-\epsilon}-\zeta_{33}^{d}\right)} \\
-\left|\eta^{\prime} / X_{d}\right| e^{i\left(\phi_{\eta+\epsilon}+\phi_{X_{d}}\right)} & e^{i\left(\phi_{\eta+\epsilon}+\phi_{X_{d}}-\zeta_{u s}\right)} & |\eta+\epsilon| e^{i\left(\phi_{\eta+\epsilon}+\zeta_{33}^{d}\right)} \\
\left|\eta^{\prime} / X_{d}\right||\eta+\epsilon| e^{i\left(\phi_{X_{d}}\right)}-Y & -|\eta+\epsilon| e^{i\left(\phi_{X_{d}}-\zeta_{u s}\right)} & e^{i \zeta_{33}^{d}}
\end{array}\right]
$$

\footnotetext{
${ }^{4}$ modulo the contribution from the strong $\mathrm{CP}$ parameter $\Theta$

${ }^{5}$ For instance, consider the superpotential for $\mathbf{4 5}_{H}$ only: $\left.\left.\mathrm{W}\left(\mathbf{4 5} \mathbf{5}_{H}\right)=M_{\mathbf{4 5}}(\mathbf{4 5})_{H}\right)^{2} " \lambda(\mathbf{4 5})_{H}\right)^{4} / M$, which yields (setting $\left.F_{\mathbf{4 5}}=0\right)$, either $\left\langle\mathbf{4 5}_{H}\right\rangle=0$, or $\left\langle\mathbf{4 5}_{H}\right\rangle^{2}=-\left(2 M_{\mathbf{4 5}} M /\right.$ " $\lambda$ " $)$. Assuming that "other physics" would favor $\left\langle\mathbf{4} \mathbf{5}_{H}\right\rangle \neq 0$, we see that $\left\langle\mathbf{4 5} \mathbf{5}_{H}\right\rangle$ would be pure imaginary, if the quantity in the brackets is positive with all parameters being real. In a coupled system, it is conceivable that $\left\langle\mathbf{4 5}_{H}\right\rangle$ in turn would induce phases (other than 0 and $\pi$ ) in some of the other VEVs as well, and may itself become complex rather than pure imaginary.
} 


$$
X_{R}^{d} \simeq\left[\begin{array}{ccc}
e^{i\left(\phi_{\eta+\epsilon}+\phi_{X_{d}}\right)} & \left|\eta^{\prime} / X_{d}\right| e^{i\left(\phi_{\eta+\epsilon}+\phi_{X_{d}}-\zeta_{u s}\right)} & \eta^{\prime}|\eta+\epsilon| e^{i\left(\phi_{\eta+\epsilon}+\zeta_{33}^{d}\right)} \\
-\left|\eta^{\prime} / X_{d}\right| e^{-i\left(\phi_{\eta-\epsilon}\right)} & e^{-i\left(\phi_{\eta-\epsilon}+\zeta_{u s}\right)} & |\eta-\epsilon| e^{-i\left(\phi_{\eta-\epsilon}-\zeta_{33}^{d}\right)} \\
\left|\eta^{\prime} / X_{d}\right||\eta-\epsilon| & -|\eta-\epsilon| e^{-i \zeta_{u s}} & e^{i \zeta_{33}^{d}}
\end{array}\right]
$$

Here $\phi_{\eta \pm \epsilon} \equiv \arg (\eta \pm \epsilon)$, that is, $\left(\eta \pm \epsilon \equiv|\eta \pm \epsilon| e^{i \phi_{\eta \pm \epsilon}}\right) ; Y \equiv \eta^{\prime}|\eta-\epsilon| e^{-i \zeta_{u d}}$ and $X_{d} \equiv-\left|\eta^{2}-\epsilon^{2}\right|+\left|\zeta_{22}^{d}\right| e^{-i\left(\phi_{\eta+\epsilon}+\phi_{\eta-\epsilon}-\phi_{\zeta_{2 d}}\right)} \equiv\left|X_{d}\right| e^{i \phi_{X_{d}}}$, where $\zeta_{22}^{d} \equiv\left|\zeta_{22}^{d}\right| e^{i \phi_{\zeta_{2 d}}}$. The corresponding matrices $X_{L, R}^{u}$ for diagonalizing the up sector are obtained from above with the substitutions $: \eta \rightarrow \sigma ; \zeta_{22}^{d} \rightarrow \zeta_{22}^{u} ;\left(\eta^{\prime} \pm \epsilon^{\prime}\right) \rightarrow \pm \epsilon^{\prime}$. Thus $\phi_{\eta \pm \epsilon}$ are replaced by $\phi_{\sigma \pm \epsilon} \equiv \arg (\sigma \pm \epsilon)$; and $X_{d}$ by $X_{u} \equiv-\left|\sigma^{2}-\epsilon^{2}\right|+\left|\zeta_{22}^{u}\right| e^{-i\left(\phi_{\sigma+\epsilon}+\phi_{\sigma-\epsilon}-\phi_{\zeta_{2 u}}\right)} \equiv\left|X_{u}\right| e^{i \phi_{X_{u}}}$. Given the definitions of $\phi_{X_{d}}$ and $\phi_{X_{u}}$ as above, we have

$$
\zeta_{33}^{d} \simeq\left(\phi_{X_{d}}-\phi_{\eta-\sigma}+\phi_{\eta+\epsilon}\right)+R \sin \Omega ; \quad \gamma \equiv\left(\phi_{\eta+\epsilon}+\phi_{\eta-\epsilon}\right)-\left(\phi_{\sigma+\epsilon}+\phi_{\sigma-\epsilon}\right)+\phi_{\epsilon^{\prime}}
$$

where

$$
\begin{aligned}
R & \equiv\left|\epsilon^{\prime} / X_{u}\right| /\left|\eta^{\prime} / X_{d}\right| \approx \sqrt{m_{u} / m_{c}} \sqrt{m_{s} / m_{d}} \approx 0.3 ; \\
\beta_{\Omega} & \approx R(\sin \Omega / \Omega), \Omega \equiv\left(\phi_{X_{d}}-\phi_{X_{u}}\right)+\gamma ; \\
\zeta_{c b} & \simeq \arg \left[e^{i\left(\gamma-\phi_{X_{u}}\right)}\left\{|\eta+\epsilon|-|\sigma+\epsilon| e^{i\left(\phi_{\sigma+\epsilon}-\phi_{\eta+\epsilon}\right)}\right\}\right] \\
\zeta_{u s} & \approx-R \sin \Omega[1-R \cos \Omega]^{-1} .
\end{aligned}
$$

As mentioned above, using observed fermion masses and mixings [9], we obtain: $\left|\epsilon^{\prime}\right| \sim$ $1 / 10\left|\eta^{\prime}\right|$, with $\left|\eta^{\prime}\right| \sim($ few $) \times 10^{-3} \ll(|\eta| \sim|\epsilon| \sim|\sigma| \sim 1 / 10) \ll 1$. In writing Eqns. (9) and (10), we have not displayed for simplicity of writing, small correction terms $\left(\mathcal{O}\left(\epsilon^{2}, \eta^{2}\right)\right)$, which are needed to preserve unitarity. We have also not displayed small phases of order $\left|\eta^{\prime} \epsilon^{\prime} / X_{u} X_{d}\right| \times \sin \Omega \sim 1 / 100,\left|\epsilon^{\prime} / \eta^{\prime}\right| \sim 1 / 10$ and $R \sin \Omega \sim 1 / 10$. Our results to be presented, that are based on exact numerical calculations, however incorporate these small corrections.

The CKM elements in the Wolfenstein basis are given by the matrix $V_{C K M}=$ $e^{-i \alpha}\left(X_{L}^{u \dagger} X_{L}^{d}\right)$, where $\alpha=\left(\phi_{\sigma-\epsilon}-\phi_{\eta-\epsilon}\right)-\left(\phi_{\epsilon^{\prime}}-\phi_{\eta^{\prime}+\epsilon^{\prime}}\right)$, where without loss of generality (given $\left|\eta^{\prime}\right| \gg\left|\epsilon^{\prime}\right|$ ), we can choose $\phi_{\eta^{\prime}+\epsilon^{\prime}} \approx 0$. To a good approximation, the CKM elements 
are given by:

$$
\begin{aligned}
& V_{u d} \approx V_{c s} \approx V_{t b} \approx 1 \\
& V_{u s} \approx|| \eta^{\prime} / X_{d}|-| \epsilon^{\prime} / X_{u}\left|e^{i \Omega}\right| \approx-V_{c d} \\
& V_{c b} \approx\left|e^{i\left(\gamma-\phi_{X_{u}}\right)}\left\{|\eta+\epsilon|-|\sigma+\epsilon| e^{i\left(\phi_{\sigma+\epsilon}-\phi_{\eta+\epsilon}\right)}\right\}\right| \approx-V_{t s} \\
& V_{u b} \approx\left[\eta^{\prime}|\eta-\epsilon|-\left|\epsilon^{\prime} / X_{u}\right| e^{i\left(\gamma-\phi_{X_{u}}\right)}\left\{|\eta+\epsilon|-|\sigma+\epsilon| e^{-i\left(\phi_{\sigma+\epsilon}-\phi_{\eta+\epsilon}\right)}\right\}\right] \times e^{i\left[\Omega\left(1+\beta_{\Omega}\right)-\zeta_{c b}\right]} \\
& V_{t d} \approx\left[\left|\eta^{\prime} / X_{d}\right| e^{i\left(\phi_{X_{d}}\right)}\left\{|\epsilon+\eta|-|\sigma+\epsilon| e^{-i\left(\phi_{\sigma+\epsilon}-\phi_{\eta+\epsilon}\right)}\right\}-\eta^{\prime}|\eta-\epsilon|\right] \times e^{-i\left[\Omega\left(1+\beta_{\Omega}\right)-\zeta_{c b}\right]}
\end{aligned}
$$

Note that the CKM elements have the desired Wolfenstein form with only $V_{u b}$ and $V_{t d}$ being complex and the others being real to a good approximation. $\zeta_{c b}$ defined above is just the argument of the expression within the bars for $V_{c b}$. One can check that to a good approximation, (neglecting the $\eta^{\prime}|\eta-\epsilon|$ term for $V_{t d}$ that causes $<10 \%$ error), the phase of $V_{t d}$ is given by $\phi_{t d} \equiv \operatorname{Arg}\left(V_{t d}\right) \approx-R \sin \Omega$, and $\left|V_{t d}\right| \approx\left|\eta^{\prime}\right| X_{d}|| V_{c b}^{*}\left|\approx \sqrt{m_{d} / m_{s}}\right| V_{c b} \mid$, and similarly $\left|V_{u b}\right| \approx \sqrt{m_{u} / m_{c}}\left|V_{c b}\right|$.

Before presenting the results of a certain fit and the corresponding predictions, we need to first discuss SUSY CP and flavor violations in the presence of phases in the fermion mass matrices. This is done in the next section.

\section{SUSY CP and Flavor Violations}

Our procedure for dealing with SUSY CP and flavor violations may be summarized by the following set of considerations:

1) As is well known, since the model is supersymmetric, non-standard CP and flavor violations would generically arise in the model through sfermion/gaugino quantum loops involving scalar $(\text { mass })^{2}$ transitions. The latter can either preserve chirality (as in $\tilde{q}_{L, R}^{i} \rightarrow$ $\tilde{q}_{L, R}^{j}$ ) or flip chirality (as in $\tilde{q}_{L, R}^{i} \rightarrow \tilde{q}_{R, L}^{j}$ ). Subject to our assumption on SUSY breaking (specified below), it would turn out that these scalar $(\text { mass })^{2}$ parameters get completely determined within our model by the fermion mass-matrices, and the few parameters of SUSY breaking.

2) SUSY Breaking : We assume that SUSY breaking is communicated to the SM sector by messenger fields which have large masses of order $M^{*}$, where $M_{G U T} \lesssim M^{*} \approx M_{\text {string}}$, such that the soft parameters are flavor-blind, and family-universal at the scale $M^{*}$. A number of well motivated models of SUSY breaking, e.g., those based on mSUGRA [18], gaugino- 
mediation [19], anomalous $U(1)-D$ term [20,21], combined with dilaton-mediation [21,22], or possibly a combination of some of these mechanisms, do in fact induce such a breaking. While for the first two cases $[18,19]$ we would expect extreme squark degeneracy (ESD) i.e. $\kappa \equiv\left|m^{2}\left(\tilde{q}_{i}\right)-m^{2}\left(\tilde{q}_{j}\right)\right| / m^{2}(\tilde{q})_{A V} \ll 10^{-3}$ (say) at the scale $M^{*}$, for the third case [20,21], one would expect intermediate squark degeneracy (ISD) i.e. $\kappa \sim 10^{-2}(1-1 / 3)$ at $M^{*}$. For the sake of generality, we would initially allow both possibilities, $\kappa=0$ (ESD), and $\kappa \sim 10^{-2}(1-1 / 3)(\mathrm{ISD})$ at $M^{*}$.

In an extreme version of universality, analogous to CMSSM, the SUSY sector of the model would introduce only five parameters at the scale $M^{*}$ :

$$
m_{o}, m_{1 / 2}, A_{o}, \tan \beta \text { and } \operatorname{sgn}(\mu)
$$

In some cases, $A_{o}$ can be zero or extremely small $(\lesssim 1 \mathrm{GeV})$ at $M^{*}$ as in [19] and [21]. For most purposes we will adopt this restricted version of SUSY breaking, including the vanishing of $A_{o}$ at $M^{*}$. However, our results will be essentially unaffected even if $A_{o}$ is nonzero ( $\sim 500 \mathrm{GeV}$, say) but real (see remarks later). We will not insist on, but will allow for, Higgs-squark-slepton universality, which does not hold, for example, in the string-derived model of [21]. In spite of flavor-preservation at a high scale $M^{*}$, SUSY-induced flavorviolation would still arise at the electroweak scale through renormalization group running of the sfermion masses and the $A$-parameters from $M^{*} \rightarrow M_{G U T} \rightarrow m_{W}$, as specified below. Although the premises of our model as regards the choice of universal SUSY parameters coincide with that of CMSSM, as we will see, owing to the presence of GUT-scale physics in the interval $M^{*} \rightarrow M_{G U T}$, SUSY CP and flavor violations in our model (evaluated at the electroweak scale) would be significantly enhanced compared to that in CMSSM (or even CMSSM with right-handed neutrinos). This difference provides some distinguishing features of our model.

\section{3) Flavor Violation due to RG Running of Scalar Masses from $\mathbf{M}^{*}$ to $\mathbf{M}_{\mathrm{GUT}}$}

For MSSM embedded into SO(10) above the GUT scale, there necessarily exist heavy color-triplet Higgs fields which couple to fermions through the coupling $h_{t} \mathbf{1 6}_{3} \mathbf{1 6}_{3} \mathbf{1 0}_{H}$, while there exist heavy doublets for both $\mathrm{SO}(10)$ and $\mathrm{G}(224)$ which also couple to fermions owing to the mixing of $\mathbf{1 0}_{H}$ with $\mathbf{1 6}_{H}$ (see [9]). (Here $h_{t}$ stands for $h_{33}$ of Eq. (4)). These couple

to $\tilde{b}_{L}$ and $\tilde{b}_{R}$ with the large top quark Yukawa coupling $h_{t}$. The heavy triplets and doublets 
possess masses of order $M_{G U T}$. One can verify (see [23]) that the evolution of RG equations for squark masses involving such couplings suppress $\tilde{b}_{L}$ and $\tilde{b}_{R}$ masses significantly compared to those of $\tilde{d}_{L, R}$ and $\tilde{s}_{L, R}$. Note that left-right symmetry implies equal shifts in $\tilde{b}_{L}$ and $\tilde{b}_{R}$ masses arising from GUT scale physics in the momentum range $M_{G U T} \leq \mu \leq M^{*}$. Such differential mass shifts i.e.- $\left(\hat{m}_{3}^{2}-\hat{m}_{1,2}^{2}\right)_{L, R} \equiv \Delta \hat{m}_{\tilde{b}_{L, R}}^{2}$, for the embedding of MSSM into $\mathrm{SO}(10)$, are found to be (with $A_{o}=0$ ):

$$
\Delta \hat{m}_{\tilde{b}_{R}}^{2}=\Delta \hat{m}_{\tilde{b}_{L}}^{2} \approx-\left(\frac{30 m_{o}^{2}}{16 \pi^{2}}\right) h_{t}^{2} \ln \left(M^{*} / M_{G U T}\right) \equiv-\left(m_{o}^{2} / 4\right) \xi .
$$

The hat signifies GUT scale values. Here $m_{o}$ denotes the (approximately) degenerate mass of squarks at the scale $M^{*}$. We have set $h_{t}^{2}=1 / 2$; we expect $M^{*} / M_{G U T} \sim(3$ to 10$)$, say, and thus, $\xi \equiv \ln \left(M^{*} / M_{G U T}\right) / 2.6 \approx(0.4$ to 0.9$)$. For the case of MSSM embedded into G(224), which provides the heavy doublet, but not the triplets, the factor 30 in Eq. (12) should be replaced by 12 .

Having diagonalized the quark mass-matrices $M_{d}^{(0)}$ and $M_{u}^{(0)}$ at the GUT scale by matrices as in Eq. (8), SUSY flavor violation may be assessed by imposing the parallel transformations on the squark $(\text { mass })^{2}$ matrices $\left(\left(\tilde{M}_{d}^{(0)}\right)_{L L / R R}\right)$ defined in the gauge basis, i.e., by evaluating $X_{L}^{d \dagger}\left(\tilde{M}_{d}^{(0)}\right)_{L L} X_{L}^{d}$ and $X_{R}^{d \dagger}\left(\tilde{M}_{d}^{(0)}\right)_{R R} X_{R}^{d}$, and similarly for the up sector. Following discussion on SUSY breaking, the off-diagonal elements (in the gauge basis) and all chirality flipping elements are set to be zero - i.e $\left(\tilde{M}_{i j}^{(0)}\right)_{L L / R R}=0(i \neq j)$ and $\left(A_{i j}^{0}\right)_{L R}=0$ - at the scale $M^{*}$. Once squarks are non degenerate at $M_{G U T}$ owing to the mass-shift of $\tilde{b}_{L, R}$ as in Eq. (12), the transformations mentioned above induce off-diagonal elements with squarks being in the SUSY basis. For the down squark mass matrices (evaluated at the GUT scale), these off diagonal elements are found to be: 


$$
\begin{aligned}
& \left.\hat{\delta}_{L L}^{12}\left(M_{G U T}\right) \simeq\left[\kappa_{I S D}^{12}+\left(\Delta \hat{m}_{\tilde{b}_{L}}^{2} / m_{s q}^{2}\right)\left(-\left|\eta^{\prime} / X_{d}\right||\epsilon+\eta|^{2}+\eta^{\prime}\left|\epsilon^{2}-\eta^{2}\right| e^{i \phi_{X_{d}}}\right)\right\}\right] e^{-i \phi_{t d}} \\
& \approx\left[\kappa_{I S D}^{12}+1.5 \times 10^{-4} \xi\right]\left(m_{o}^{2} / m_{s q}^{2}\right) e^{-i \phi_{t d}} \\
& \left.\hat{\delta}_{R R}^{12}\left(M_{G U T}\right) \simeq\left[\kappa_{I S D}^{12}+\left(\Delta \hat{m}_{\tilde{b}_{R}}^{2} / m_{s q}^{2}\right)\left(-\left|\eta^{\prime} / X_{d}\right||\epsilon-\eta|^{2}+\eta^{\prime}\left|\epsilon^{2}-\eta^{2}\right| e^{-i \phi_{X_{d}}}\right)\right\}\right] e^{-i \phi_{t d}} \\
& \approx\left[\kappa_{I S D}^{12}+3 \xi \times 10^{-3}-10^{-5}(\xi) e^{-i \phi_{X_{d}}}\right]\left(m_{o}^{2} / m_{s q}^{2}\right) e^{-i \phi_{t d}} \\
& \hat{\delta}_{L L}^{13}\left(M_{G U T}\right) \simeq\left(\Delta \hat{m}_{\tilde{b}_{L}}^{2} / m_{s q}^{2}\right)\left[-\eta^{\prime}|\eta-\epsilon| e^{i \zeta_{33}^{d}}+\left|\eta^{\prime} / X_{d}\right||\eta+\epsilon| e^{i\left(\zeta_{33}^{d}-\phi_{X_{d}}\right)}\right] \\
& \approx\left[(2.5 \xi) \times 10^{-4} e^{i \zeta_{33}^{d}}-(2.5 \xi) \times 10^{-3} e^{i\left(\zeta_{33}^{d}-\phi_{X_{d}}\right)}\right]\left(m_{o}^{2} / m_{s q}^{2}\right) \\
& \hat{\delta}_{R R}^{13}\left(M_{G U T}\right) \simeq\left(\Delta \hat{m}_{\tilde{b}_{R}}^{2} / m_{s q}^{2}\right)\left[-\eta^{\prime}|\eta+\epsilon| e^{i\left(\zeta_{33}^{d}-\phi_{X_{d}}\right)}+\left|\eta^{\prime} / X_{d}\right||\eta-\epsilon| e^{i \zeta_{33}^{d}}\right] \\
& \approx-\left[(1.25 \xi) \times 10^{-2} e^{i \zeta_{33}^{d}}\right]\left(m_{o}^{2} / m_{s q}^{2}\right) \\
& \hat{\delta}_{L L}^{23}\left(M_{G U T}\right) \simeq\left(\Delta \hat{m}_{\tilde{b}_{L}}^{2} / m_{s q}^{2}\right)\left[-|\eta+\epsilon| e^{i\left(\zeta_{33}^{d}-\phi_{X_{d}}+\phi_{t d}\right)}\right] \\
& \approx\left[(1.25 \xi) \times 10^{-2} e^{i\left(\zeta_{33}^{d}-\phi_{X_{d}}+\phi_{t d}\right)}\right]\left(m_{o}^{2} / m_{s q}^{2}\right) \\
& \hat{\delta}_{R R}^{23}\left(M_{G U T}\right) \simeq\left(\Delta \hat{m}_{\tilde{b}_{R}}^{2} / m_{s q}^{2}\right)\left[-|\eta-\epsilon| e^{i\left(\zeta_{33}^{d}+\phi_{t d}\right)}\right] \\
& \approx\left[(6.2 \xi) \times 10^{-2} e^{i\left(\zeta_{33}^{d}+\phi_{t d}\right)}\right]\left(m_{o}^{2} / m_{s q}^{2}\right)
\end{aligned}
$$

Here $\kappa_{I S D}^{12} \equiv\left[\left(m_{1}^{(0) 2}-m_{2}^{(0) 2}\right)\right] / m_{s q}^{2}\left(\left|\eta^{\prime} / X_{d}\right|\right) \sim \pm\left(2 \times 10^{-3}\right)(1-1 / 3) ;$ this term would be present for the case of intermediate squark degeneracy (ISD), corresponding to small $\left(\sim 10^{-2}(1-1 / 3)\right)$ squark non-degeneracy at the scale $M^{*}$, as in models of Ref. [20,21]. From now on, for the sake of concreteness, we drop this term, ${ }^{6}$ setting $\kappa_{I S D}^{12}=0$. In above $\phi_{t d} \approx$ $-\left|\epsilon^{\prime} / X_{u}\right| /\left|\eta^{\prime} / X_{d}\right| \sin \Omega \sim(-1 / 3) \sin \Omega \sim(-1 / 6)$ (say). The hat on top signifies GUT scale values, and $\hat{\delta}_{L L / R R}^{i j} \equiv\left(\hat{\Delta}_{L L / R R}^{i j}\right) / m_{s q}^{2}$, where $\hat{\Delta}_{L L}^{i j}$ denotes the $(\text { mass })^{2}$ parameter for $\tilde{q}_{j L} \rightarrow \tilde{q}_{i L}$ transition in the SUSY basis. Here, $m_{s q}$ denotes the average mass of the $\tilde{d}_{L, R}$ and $\tilde{s}_{L, R}$ squarks, which remain nearly degenerate( to $1 \%$ or better) even at the weak scale. For each $\hat{\delta}_{L L / R R}^{i j}$ we have exhibited approximate numerical values by inserting values of the parameters $\eta, \sigma, \epsilon \eta^{\prime}$ etc. for some typical fits (as in Eqs. (6) and (201)) to indicate their typical values.

\footnotetext{
${ }^{6}$ Note that the case of ISD $\left(\kappa_{I S D}^{12} \sim\left(2 \times 10^{-3}\right)(1-1 / 3) \neq 0\right)$ would make a difference only for the case of $K^{o}-\overline{K^{o}}$ transitions - that is, for $\Delta m_{K}$ and $\epsilon_{K}$.
} 
Assuming for simplicity, universality of scalar masses $m_{o}$ (of the first two families) and of the gaugino masses $m_{1 / 2}$ at the GUT scale, the physical masses of squarks of the first two families and of the gluino are given by:

$$
m_{s q}^{2} \approx m_{o}^{2}+7.2 m_{1 / 2}^{2} ; \quad m_{\tilde{g}} \approx 2.98 m_{1 / 2}
$$

This result is rather insensitive to the mass shifts of $\tilde{b}_{L, R}$. Using the above relations we get $\rho_{X} \equiv\left(m_{o}^{2} / m_{s q}^{2}\right) \simeq 1-0.8 x \approx(0.84,0.76,0.5$ and 0.2$)$ for $x \equiv m_{\tilde{g}}^{2} / m_{s q}^{2}=(0.2,0.3,0.6$ and 1$)$, which enters into all the $\hat{\delta}^{i j}$-elements in Eq. (13).

We remind the reader that the elements $\hat{\delta}_{L L, R R}^{i j}$, induced solely through GUT scale physics being relevant in the interval $M^{*} \rightarrow M_{G U T}$, would be absent in a general CMSSM or MSSM, and so would the associated CP and flavor violations.

4) Flavor Violation Through RG Running From $\mathbf{M}_{\mathrm{GUT}}$ to $\mathbf{m}_{\mathrm{W}}$ in MSSM : It is well known that, even with universal masses at the GUT scale, RG running from $M_{G U T}$ to $m_{W}$ in MSSM, involving contribution from the top Yukawa coupling, gives a significant correction to the mass of $\tilde{b}_{L}^{\prime}=V_{t d} \tilde{d}_{L}+V_{t s} \tilde{s}_{L}+V_{t b} \tilde{b}_{L}$, which is not shared by the mass-shifts of $\tilde{b}_{R}, \tilde{d}_{L, R}$ and $\tilde{s}_{L, R}$. This in turn induces flavor violation. Here, $\tilde{d}_{L}, \tilde{s}_{L}$ and $\tilde{b}_{L}$ are the SUSY partners of the physical $d_{L}, s_{L}$ and $b_{L}$ respectively. The differential mass shift of $\tilde{b}_{L}^{\prime}$ arising as above, may be expressed by an effective Lagrangian [24]: $\Delta \mathcal{L}=-\left(\Delta m_{L}^{\prime 2}\right) \tilde{b}_{L}^{\prime} \tilde{b}_{L}^{\prime}$, where ${ }^{7}$

$$
\Delta m_{L}^{\prime 2}=-3 / 2 m_{o}^{2} \eta_{t}+2.3 A_{o} m_{1 / 2} \eta_{t}\left(1-\eta_{t}\right)-\left(A_{o}^{2} / 2\right) \eta_{t}\left(1-\eta_{t}\right)+m_{1 / 2}^{2}\left(3 \eta_{t}^{2}-7 \eta_{t}\right)
$$

Here $\eta_{t}=\left(h_{t} / h_{f}\right) \approx\left(m_{t} / v \sin \beta\right)^{2}(1 / 1.21) \approx 0.836$ for $\tan \beta=3$. Numerically, setting $^{8}$ $A_{o}=0$, Eq. (15) yields: $\left(\Delta m_{L}^{\prime 2} / m_{s q}^{2}\right) \approx-(0.40,0.34,0.26,0.22)$ for $x=m_{\tilde{g}}^{2} / m_{s q}^{2} \approx$ $(0.1,0.4,0.8,1.0)$. Expressing $\tilde{b}_{L}^{\prime}$ in terms of down-flavor squarks in the SUSY basis as above, Eq. (15) yields new contributions to off diagonal squark mixing. Normalizing to $m_{s q}^{2}$, they are given by

$$
\delta_{L L}^{\prime(12,13,23)}=\left(\frac{\Delta m_{L}^{\prime 2}}{m_{s q}^{2}}\right)\left(V_{t d}^{*} V_{t s}, \quad V_{t d}^{*} V_{t b}, V_{t s}^{*} V_{t b}\right)
$$

The net squark (mass) ${ }^{2}$ off-diagonal elements at $m_{W}$ are then obtained by adding the

\footnotetext{
${ }^{7}$ Note that strictly speaking Eq. (15) holds if the soft parameters are universal at the GUT-scale. However, the correction to this expression due to RG running from $M^{*}$ to $m_{W}$ would be rather small, being a correction to a correction.

${ }^{8}$ Although we have put $A_{o}=0$ (for concreteness), note that $\Delta m_{L}^{\prime 2}$ would typically get only a small correction ( $\lesssim 5 \%$ ), even if $A_{o}$ were non-zero $(\lesssim 1 \mathrm{TeV})$, with $m_{o} \approx(0.7-1) \mathrm{TeV}$ and $m_{1 / 2} \approx(200-300) \mathrm{GeV}$, say.
} 
respective GUT-scale contributions from Eqs. (13) to that from Eq. (16). They are:

$$
\delta_{L L}^{i j}=\hat{\delta}_{L L}^{i j}+\delta_{L L}^{\prime i j} ; \quad \delta_{R R}^{i j}=\hat{\delta}_{R R}^{i j}
$$

From the expressions given above (Eqs. (13) and (16)), it follows that for a given choice of the SUSY-parameters (i.e. $m_{o}, m_{1 / 2}$ or equivalently $m_{s q}$ and $m_{\tilde{g}}$ ), SUSY CP and flavor violations are completely determined within our model by parameters of the fermion massmatrices. This is the reason why within a quark-lepton unified theory as ours, SUSY CP and flavor violations get intimately related to fermion masses and neutrino oscillations.

5) A - Terms Induced Through RG Running from $\mathbf{M}^{*}$ to $\mathbf{M}_{\mathrm{GUT}}$ : Even if $A_{o}$ is zero at $M^{*}$ (as we assume, for concreteness, see also [19] and [21]), RG running from $M^{*}$ to $M_{G U T}$ in the context of $\mathrm{SO}(10) / \mathrm{G}(224)$ would still induce non-zero $A$ parameters at the GUT scale [23]. For our case, the $A$ terms are induced through loop diagrams involving the $h_{33}, g_{23}$, and $a_{23}$ couplings and the $\mathrm{SO}(10)$ or $\mathrm{G}(224)$ gauginos. We find that if we take $M_{10_{H}} \approx M_{16_{H}} \approx M_{G U T}$, we can write the $A_{L R}$-matrix at the GUT-scale for the down squark sector in the SUSY basis for the case of $\mathrm{SO}(10)$ as follows:

$$
A_{L R}^{d}=Z \times\left(X_{L}^{d}\right)^{\dagger}\left[\begin{array}{ccc}
0 & 95 \epsilon^{\prime}+90 \eta^{\prime} & 0 \\
-95 \epsilon^{\prime}+90 \eta^{\prime} & 63 \zeta_{22}^{d} & 95 \epsilon+90 \eta-27 \sigma \\
0 & -95 \epsilon+90 \eta-27 \sigma & 63
\end{array}\right] X_{R}^{d}
$$

where $Z=\left(\frac{1}{16 \pi^{2}}\right) h_{t} g_{10}^{2} M_{\lambda} \ln \left(\frac{M^{*}}{M_{G U T}}\right)$, and $\left(X^{d}\right)_{L, R}$ are given in Eqs. (19) and (10). The $g_{23}$ coupling does not contribute to the up-sector; thus the $A$-matrix for the up squarks, $A_{L R}^{u}$, can be obtained from above by setting $\eta^{\prime}=0$ and replacing $90 \eta-27 \sigma$ by $63 \sigma, \zeta_{22}^{d}$ by $\zeta_{22}^{u}$, and $X_{L, R}^{d}$ by $X_{L, R}^{u}$ in $A^{d}$. Similarly, the lepton $A$-matrix, $A_{L R}^{l}$ is obtained by letting $\left(\epsilon, \epsilon^{\prime}\right) \rightarrow-3\left(\epsilon, \epsilon^{\prime}\right)$ and replacing $X_{L, R}^{d}$ by $X_{L, R}^{l}$ in $A_{L R}^{d}$. For the case of $\mathrm{G}(224)$, the matrix $A_{L R}^{d}$ would be obtained by making the substitutions: $(90,63,95) \rightarrow(42,27,43)$ in Eq. (18), and likewise in $A_{L R}^{u}$ and $A_{L R}^{l}$. It is sometimes convenient to define the sfermion transition mixing angles as

$$
\left(\delta_{L R}^{d, l}\right)_{i j} \equiv\left(A_{L R}^{d, l}\right)_{i j}\left(\frac{v_{d}}{m_{s q}^{2}}\right)=\left(A_{L R}^{d, l}\right)_{i j}\left(\frac{v_{u}}{\tan \beta m_{s q}^{2}}\right) ; \quad\left(\delta_{L R}^{u}\right)_{i j} \equiv\left(A_{L R}^{u}\right)_{i j}\left(\frac{v_{u}}{m_{s q}^{2}}\right) .
$$

Note that these induced $A_{L R^{-}}$-terms for all three sectors, like the squarks (mass) ${ }^{2}$ elements $\delta_{L L, R R}^{i j}$ given in Eqs. (13)-(17), are completely determined within our model by the fermion 
mass matrices, for a given choice of $M_{\lambda} \approx m_{1 / 2}$ and $\ln \left(M^{*} / M_{G U T}\right)$. We now utilize these SUSY CP and flavor-violating elements to predict the results of our model.

Once again, as in the case of $\hat{\delta}_{L L, R R}^{i j}$, these induced $A$-terms arising purely through GUTphysics, would be absent or negligibly small in CMSSM. As a result, some of the interesting predictions of our model as regards $\epsilon_{K}^{\prime}$ and edm's (to be discussed below) and lepton flavor violations (to be discussed in a forthcoming paper [25]) would be absent altogether in CMSSM.

\section{Compatibility of CP and Flavor Violations with Fermion Masses and Neutrino Oscillations in $\mathrm{SO}(10) / \mathrm{G}(224)$ : Our Results}

It has been noted in Sec. 1 that (given about 15\% uncertainty in the matrix elements) the SM agrees very well with all four entries of Eq. (1D), for a single choice of the Wolfenstein parameters $\bar{\rho}_{W}$ and $\bar{\eta}_{W}$ (Eq. (22)). The question then arises (as noted in Sec. 1): If a SUSY $\mathrm{SO}(10)$ or $\mathrm{G}(224)$ model is constrained by requiring that it should successfully describe fermion masses and neutrino oscillations (as in Sec. 2), can it still yield (for some choice of phases in the parameters $\eta, \sigma, \epsilon$ etc.) values for $\bar{\rho}_{W}$ and $\bar{\eta}_{W}$ more or less in accord with the SM-based phenomenological values for the same, as listed in Eq. (2)? Anticipating that (for any given choice of the parameters $\eta, \sigma, \epsilon$ etc.) the $\mathrm{SO}(10) / \mathrm{G}(224)$ model-based values of $\bar{\rho}_{W}$ and $\bar{\eta}_{W}$ would generically differ from the SM-based phenomenological values (given in Eq.(21)), we will denote the former by $\bar{\rho}_{W}^{\prime}$ and $\bar{\eta}_{W}^{\prime}$ and the corresponding contributions from the SM-interactions (based on $\bar{\rho}_{W}^{\prime}$ and $\bar{\eta}_{W}^{\prime}$ ) by $S M^{\prime}$. The question that faces us then is this: When the $S M^{\prime}$ contributions are added to the SUSY contributions arising from the three sources listed in Sec. 4, can such a constrained $\mathrm{SO}(10)$ or $\mathrm{G}(224)$ model account for the observed values of all the four quantities listed in Eq. (11), and in addition is it consistent with the empirical upper limits on the edm's of the neutron and the electron?'.

Before presenting our results, we make some preliminary remarks. First of all one might have thought, given the freedom in the choice of phases in the parameters of the mass matrices, that it ought to be possible to get almost any set of values of $\left(\bar{\rho}_{W}\right.$ and $\left.\bar{\eta}_{W}\right)$, and in particular those in accord with the SM values (Eq. (2)). It turns out, however, that in

\footnotetext{
${ }^{9}$ We extend the same question to include lepton flavor violating processes (such as $\mu \rightarrow e \gamma$ and $\tau \rightarrow \mu \gamma$ ) in a separate note.
} 
general this is indeed not possible without running into a conflict with the fermion masses and/or neutrino oscillation parameters within a $\mathrm{SO}(10)$ or $\mathrm{G}(224)$-model ${ }^{10}$. In other words, any predictive $\mathrm{SO}(10)$ or $\mathrm{G}(224)$-model is rather constrained in this regard.

Second, one might think that even if the $\mathrm{SO}(10) / \mathrm{G}(224)$ model-derived entities $\bar{\rho}_{W}^{\prime}$ and $\bar{\eta}_{W}^{\prime}$, constrained by the pattern of fermion masses and neutrino oscillations, are found to be very different in signs and/or magnitudes from the SM values shown in Eq. (2), perhaps the SUSY contributions added to the $S M^{\prime}$ contributions(based on $\bar{\rho}_{W}^{\prime}$ and $\bar{\eta}_{W}^{\prime}$ ) could possibly account for all four quantities listed in Eq. (11). It seems to us, however, that this is simply not a viable and natural possibility, unless one is willing to invoke MSSM and finely adjust its arbitrary (in general some 105) parameters, as needed. In the latter case, the good agreement between experiments and the SM predictions would appear to be fortuitous (see Sec. 1).

This is why it seems to us that the only viable and natural solution for any SUSY G(224) or $\mathrm{SO}(10)$ model for fermion masses and neutrino oscillations is that the model, allowing for phases in the fermion mass matrices, should not only yield the masses and mixings of all fermions including neutrinos in accord with observations (as in Sec. 2), but it should yield $\bar{\rho}_{W}^{\prime}$ and $\bar{\eta}_{W}^{\prime}$ that are close to the SM values shown in Eq. (2). This, if achievable, would be a major step in the right direction. One then needs to ask: how does the combined $\left(S M^{\prime}+\mathrm{SUSY}\right)$ contributions fare for such a solution as regards its predictions for the four quantities of Eq. (11) and other CP and/or flavor violating processes, for any given choice of the SUSY parameters $\left(m_{o}, m_{1 / 2}, A_{o}, \tan \beta\right.$ and $\left.\operatorname{sgn}(\mu)\right)$ ? It should be stressed here that even if the CKM elements including $\bar{\rho}_{W}^{\prime}$ and $\bar{\eta}_{W}^{\prime}$ should turn out to be close to the SM values (Eq. (2)), the SUSY contributions can in general still have a marked effect, in accord with observations, at least on some of the processes where the $S M$ (or $\left.S M^{\prime}\right)$-contributions are naturally suppressed (as in the case for $\epsilon_{K}$, edm's and lepton flavor violating transitions). Study of these processes, some of which we discuss below, can help distinguish between the SM versus the SUSY SO(10)/G(224)-models.

Without further elaboration, we now present our main results. In this paper we will present only one fit to the parameters which has the desired properties. ${ }^{11}$ Allowing for phases $(\sim 1 / 10$ to $1 / 2)$ in the parameters $\eta, \sigma, \epsilon^{\prime}$, and $\zeta_{22}^{d}$ of the $\mathrm{G}(224) / \mathrm{SO}(10)$ framework (see

\footnotetext{
${ }^{10}$ for a discussion of difficulties in this regard within a recently proposed $\mathrm{SO}(10)$ model, see e.g. [26]

${ }^{11}$ We have verified that there actually exists a class of fits which nearly serve the same purpose. Only one of these (Eq. 20 ) is exhibited here for the sake of concreteness.
} 
Eq. (6) ) we find that there do exist solutions which yield masses and mixings of quarks and leptons, in accord with observations to within 10\% for most part (see discussion below), and at the same time yield $\bar{\rho}_{W}^{\prime}$ and $\bar{\eta}_{W}^{\prime}$ close to the SM values, as given in Eq. (2). A desired fit to the parameters is given by:

$$
\begin{aligned}
& \sigma=0.109-0.012 i, \quad \eta=0.122-0.0464 i, \quad \epsilon=-0.103, \quad \eta^{\prime}=2.4 \times 10^{-3}, \\
& \epsilon^{\prime}=2.35 \times 10^{-4} e^{i\left(69^{\circ}\right)}, \quad \zeta_{22}^{d}=9.8 \times 10^{-3} e^{-i\left(149^{\circ}\right)}, \quad\left(\mathcal{M}_{u}^{0}, \quad \mathcal{M}_{d}^{0}\right) \approx(100,1.1) \mathrm{GeV} .
\end{aligned}
$$

For the sake of simplicity and economy, we have set $\zeta_{22}^{u}=0$ in this fit; however, values of $\left|\zeta_{22}^{u}\right| \lesssim 10^{-3}$ can lead to similar results. Note that the magnitudes of the real parts of $\eta, \sigma, \epsilon$, and $\epsilon^{\prime}$ are nearly the same as those given in the CP-conserving case [9] (see Eq. (66)); in particular the relative signs of these real parts are identical. The fit shown above leads to the following values for the fermion masses and mixings, while preserving the predictions for the neutrino system as in Eq. (7):

$$
\begin{aligned}
& \left(m_{t}^{\text {phys }}, m_{b}\left(m_{b}\right), m_{\tau}\right) \approx(174,4.97,1.78) \mathrm{GeV} \\
& \left(m_{c}\left(m_{c}\right), m_{s}(1 G e V), m_{\mu}\right) \approx(1320,101,109) \mathrm{MeV} \\
& \left(m_{u}^{\circ}(1 G e V), m_{d}^{\circ}(1 G e V), m_{e}^{\circ}\right) \approx(10.1,3.7,0.13) \mathrm{MeV} \\
& \left(V_{u s}, V_{c b},\left|V_{u b}\right|,\left|V_{t d}\right|\right)\left(\leq m_{Z}\right) \approx(0.2250,0.0412,0.0037,0.0086) \\
& \bar{\rho}_{W}^{\prime}=0.150, \quad \bar{\eta}_{W}^{\prime}=0.374
\end{aligned}
$$

In obtaining the fermion masses at the low scales, we have not directly used $\mathcal{M}_{u}^{0}$ and $\mathcal{M}_{d}^{0}$ of Eq. (3). Instead, we have used: (a) $m_{t}\left(m_{t}\right)=167 \mathrm{GeV}$ and $m_{\tau}\left(m_{\tau}\right)=1.777 \mathrm{GeV}$ as inputs; (b) the GUT-scale predictions of our model for the ratios of masses - such as $m_{b} / m_{\tau}, m_{u, c} / m_{t}, m_{d, s} / m_{b}, m_{\mu} / m_{\tau}$ etc; (c) renormalization in 2-loop QCD of these ratios in going from the GUT-scale to an effective SUSY-scale $M_{S}=500 \mathrm{GeV}$; and (d) the evolutions in 3-loop QCD and 1-loop QED of individual fermion masses as the effective momentum runs from $M_{S}$ to the appropriate low energy scales $[27]^{12}$.

The primes on $\bar{\rho}_{W}^{\prime}$ and $\bar{\eta}_{W}^{\prime}$ signify that these values are obtained from the $\mathrm{SO}(10) / \mathrm{G}(224)$ model based fermion mass matrices (as in Eq. (3)), in conjunction with fermion masses and neutrino oscillations, as opposed to SM-based phenomenological values (Eq. (2)).

\footnotetext{
${ }^{12}$ Defining $\eta_{a / b} \equiv\left(m_{a} / m_{b}\right)_{G U T} /\left(m_{a} / m_{b}\right)_{M_{S}}$ and $\eta_{f} \equiv m_{f}\left(M_{S}\right) / m_{f}\left(\mu_{l o w}\right)$, we get (for $\tan \beta=5$ and $\left.\alpha_{3}\left(M_{Z}\right)=0.118\right)$ : $\eta_{b / \tau}=0.6430, \eta_{u, c / t}=0.4456, \eta_{d, s / b}=0.7660, \eta_{e, \mu / \tau}=0.9999, \eta_{u}=0.3954, \eta_{d, s}=0.3982, \eta_{c}=0.4418, \eta_{b}=$ $0.6053, \eta_{e, \mu}=0.9894, \eta_{\tau}=0.9914, \eta_{t}=0.9427$. The CKM elements at low scales are given by $V_{\alpha \beta}\left(\leq m_{Z}\right)=V_{\alpha \beta}(G U T) / K_{\alpha \beta}$, where $K_{\alpha \beta} \approx 0.91$ for $\alpha \beta=u b, c b, t d$, and ts and $K_{\alpha \beta} \approx 1$ for the other elements.
} 
Note that, except for the very light fermion masses $\left(m_{u}^{\circ}, m_{d}^{\circ}\right.$, and $\left.m_{e}^{\circ}\right)$ which would need corrections of order 1 to few $\mathrm{MeV}$ [28], all the other quark-lepton masses and especially the CKM mixings are in good accord with observations (see values quoted below Eq. (2) or Ref. [29]), to within 10\%. (As alluded to before, we should not of course expect the very light fermion masses to be described adequately by the gross pattern of the mass-matrices exhibited in Sec. 2. In particular the "11" entries in Eq. (3) (expected to be of order $10^{-4}-10^{-5}$ ) arising from higher dimensional operators, which have been neglected in Sec. 2, can quite plausibly lead to a needed reduction in $m_{u}$ by about $6-8 \mathrm{MeV}$ and an increase in $\left(m_{e} \text { and } m_{d}\right)^{13}$ by nearly $(0.36$ and $2-3) \mathrm{MeV}$ respectively, at the $1 \mathrm{GeV}$ scale, without altering the CKM mixings).

The important point is that the $\mathrm{SO}(10) / \mathrm{G}(224)$-model presented in Sec. 3 has turned out to be capable of yielding values for $\bar{\rho}_{W}^{\prime}$ and $\bar{\eta}_{W}^{\prime}$ that are close to the SM values as desired, while simultaneously being able to yield fermion masses of the two heavy families, all the CKM elements and neutrino oscillations (see Sec. 2), in good accord with observations. This in itself is non-trivial.

Before presenting the results for $\mathrm{CP}$ and flavor violations some comments are in order as regards the parameters of the model versus its predictiveness. As expected, introduction of (in general four) phases in the Dirac mass matrices clearly increase the number of parameters compared to that for the CP-conserving case [9]. As a result, as long as we confine to the realm consisting of (a) the fermion masses and mixings, (b) CP and flavor violations induced only by the SM interactions, and (c) neutrino oscillations, the predictiveness of the model is reduced considerably (compare with the CP-conserving case of Ref. [9], see Sec. 2), the number of parameters now being comparable to the number of observables. Nevertheless, some gross features of the predictions in fact survive, even in the realm mentioned above, simply because: (a) the entries in the mass-matrices, governed by flavor symmetries, are hierarchical with a pattern as in Eq. (3); ; (b) the phases are constrained ${ }^{14}$ to lie between 0 to $2 \pi$, and, (c) the system itself is constrained by the group theory of $\mathrm{SO}(10) / \mathrm{SU}(4)^{c}$. One

\footnotetext{
${ }^{13}$ Note that the "11" entry for the up sector can differ from that for the down sector even in sign because of contribution through the operator $\mathbf{1 6}_{1} \mathbf{1 6}_{1} \mathbf{1 6}_{H}^{d}\left(\mathbf{1 6}_{H} / M^{\prime \prime}\right)(S / M)^{n}$ which contributes only to $m_{d}$ and $m_{e}$ (so that $\delta m_{d}=\delta m_{e}$ at $M_{G U T}$ ) but not to $m_{u}$.

${ }^{14}$ For instance, consider the familiar relation $\left|V_{u s}\right|=\left|\sqrt{m_{d} / m_{s}}-e^{i \phi} \sqrt{m_{u} / m_{c}}\right|$, that holds for a hierarchical pattern. Given $\sqrt{m_{d} / m_{s}} \approx 0.22$ and $\sqrt{m_{u} / m_{c}} \approx 0.07$, we cannot of course predict $V_{u s}$ precisely without knowing the phase angle $\phi$. Yet, since $\phi$ can vary only between 0 to $2 \pi,\left|V_{u s}\right|$ must lie between 0.15 and 0.29
} 
can argue that these features in turn pretty much ensure the gross nature of the following predictions: (i) $m_{b}^{\circ} / m_{\tau}^{\circ} \approx 1$, (ii) $\left|V_{u s}\right| \sim 0.2$, (iii) $\left|V_{u b}\right| \approx \sqrt{m_{u} / m_{c}}\left|V_{c b}\right|$, (iv) $\left|V_{t d}\right| \approx$ $\sqrt{m_{d} / m_{s}}\left|V_{c b}\right|,(\mathrm{v}) m_{\nu_{2}} / m_{\nu_{3}} \sim 1 / 10$, (vi) $m_{\nu_{3}} \sim 1 / 10 \mathrm{eV}$, and, (vii) $\sin ^{2} 2 \theta_{\nu_{\mu} \nu_{\tau}}^{\text {osc }} \approx(0.8-0.99$ ), despite large variations in the parameters.

The real virtue of the model (including the phases) emerges, once one includes SUSY CP and flavor violations. In this case, the realm of observables and thereby the predictiveness of the model expands enormously. The set of observables now includes not only the four entities listed in Eq. (11)-i.e., $\Delta m_{K}, \epsilon_{K}, \Delta m_{B_{d}}$ and $S\left(B_{d} \rightarrow J / \psi K_{S}\right)$ - but also a host of others, for which the predictions of the $\mathrm{G}(224) / \mathrm{SO}(10)$ model including ( $S M^{\prime}+\mathrm{SUSY}$ ) contributions, can a priori differ significantly from those of the SM contributions. In particular, the set includes observables such as (v) $\epsilon_{K}^{\prime}$ (vi) $\Delta m_{B_{s}}$, (vii) $S\left(B_{d} \rightarrow \phi K_{S}\right)$, (viii) $S\left(B_{d} \rightarrow \eta^{\prime} K_{S}\right)$, (ix) $S\left(B_{s} \rightarrow J / \psi \phi\right),(\mathrm{x}) S\left(B_{s} \rightarrow \phi K_{S}\right)$, (xi) $B \rightarrow K \pi$, (xii) $B \rightarrow \pi \pi$ (rates and asymmetry parameters), (xiii) $b \rightarrow s \gamma$, (xiv) electric dipole moments of $(n, e, H g, d)$ and (xv) Lepton flavor violating processes $(\mu \rightarrow e \gamma, \tau \rightarrow \mu \gamma, \tau \rightarrow e \gamma)$, and more.

Now, the SUSY contributions do of course depend in part on the flavor preserving SUSYparameters (i.e. $m_{o}, m_{1 / 2}, \mu$, and $\tan \beta$; we set $A_{o}=0$ at $M^{*}$ ). But these few parameters should be regarded as extraneous to the present model, and hopefully, they would be determined through the discovery of SUSY at the LHC. The interesting point is that for a given choice of these flavor-preserving SUSY parameters (essentially $m_{o}$ and $m_{1 / 2}$ ) the SUSY contributions to all the $\mathrm{CP}$ and/or flavor-violating processes listed above get completely determined within our model, in magnitude as well as in phases. This is because all the flavor and in general CP violating sfermion $(\text { mass })^{2}$-parameters ${ }^{15}\left(\left(\delta m^{2}\right)_{L L, R R, L R}^{i j}\right)$, arising through $\mathrm{SO}(10) / \mathrm{G}(224)$-based RG running from $M^{*}$ to $M_{G U T}$ are completely fixed in the model in terms of the parameters of the fermion mass-matrices (see Eqs. (13), (18) and (19)). The latter are, however essentially fixed by fermion masses and mixings, as shown in the fit given above (Eq. (20) ), especially when we demand that the $\bar{\rho}_{W}^{\prime}$ and $\bar{\eta}_{W}^{\prime}$ be close to the SM-values. In short, the inclusion of SUSY CP and flavor violations, treated in conjunction with fermion masses and neutrino oscillations, encompasses a host of processes without introducing new parameters and thereby increases the predictiveness of the model enormously.

In this paper, we will present the results for some of the processes listed above, in par-

\footnotetext{
${ }^{15}$ counting the number of such $(\text { mass })^{2}$-parameters
} 
ticular those shown in Eq. (11) as well as those for $\epsilon_{K}^{\prime}$ and the edm's of the neutron and the electron. Some of the other processes including lepton flavor violation will be considered in a separate paper.

Using Eqs. (13) and (15)-(19) for the squarks (mass) $)^{2}$ elements ( $\delta_{L L, R R, L R}^{i j}$ etc.) as predicted in our model, the expressions given in Refs. [30-33] for the SUSY contributions, and the values of $m_{s}, m_{c}$ and the CKM elements (including $\bar{\rho}_{W}^{\prime}$ and $\bar{\eta}_{W}^{\prime}$ ) as obtained in the fit given above (see Eqs. (20) and (21) ), we can now derive the values of the four entities listed in Eq. (11), treating separately the cases of the $\mathrm{SO}(10)$ and the $\mathrm{G}(224)$-models. For reasons explained below Eq. (12), the SUSY contributions are reduced (in most cases) by about a factor of 2.5 in the amplitude for the case of $\mathrm{G}(224)$ compared to that of $\mathrm{SO}(10)$, being the effective symmetry in $4 \mathrm{D}$. This distinction, as we will see, provides a way to distinguish between the $\mathrm{SO}(10)$ and the $\mathrm{G}(224)$-models experimentally. The predictions of the model (corresponding to the fit shown in Eq. (201) ) are shown in table 1. We have included both the $S M^{\prime}$ and the SUSY contributions in obtaining the total contributions (denoted by "Tot"). In quoting the numbers we have fixed, for concreteness, $M^{*} / M_{G U T} \approx 3$ and thus $\xi \approx 0.4$, and have made a plausible choice for the SUSY spectrum - i.e. $m_{s q} \approx(0.8-1) \mathrm{TeV}$ with $x=m_{\tilde{g}}^{2} / m_{s q}^{2} \approx 0.8$, although a variation in these parameters with $m_{s q}$ as low as about 600 $\mathrm{GeV}$ or $x=0.5-0.6$ can still lead to the desired results for all four quantities especially for the case of $\mathrm{G}(224)$ (see remarks below).

\begin{tabular}{|c|c|c|c|c|}
\hline$\left(m_{o}, m_{1 / 2}\right)(\mathrm{GeV})$ & \multicolumn{2}{|c|}{$(800,250)$} & \multicolumn{2}{c|}{$(600,300)$} \\
\hline & $\begin{array}{c}(\mathrm{a}) \\
\mathrm{SO}(10)\end{array}$ & $\begin{array}{c}(\mathrm{b}) \\
\mathrm{G}(224)\end{array}$ & $\begin{array}{c}(\mathrm{c}) \\
\mathrm{SO}(10)\end{array}$ & $\begin{array}{c}\mathrm{G}(224) \\
\end{array}$ \\
\hline$\Delta m_{K}^{s . d .}\left(\mathrm{Tot} \approx S M^{\prime}\right)(\mathrm{GeV})$ & $2.9 \times 10^{-15}$ & $2.9 \times 10^{-15}$ & $2.9 \times 10^{-15}$ & $2.9 \times 10^{-15}$ \\
\hline$\epsilon_{K}\left(S M^{\prime}\right)$ & $2.83 \times 10^{-3}$ & $2.83 \times 10^{-3}$ & $2.83 \times 10^{-3}$ & $2.83 \times 10^{-3}$ \\
\hline$\epsilon_{K}(T o t)$ & $1.30 \times 10^{-3}$ & $2.32 \times 10^{-3}$ & $2.01 \times 10^{-3}$ & $2.56 \times 10^{-3}$ \\
\hline$\Delta m_{B_{d}}\left(\mathrm{Tot} \approx S M^{\prime}\right)(\mathrm{GeV})$ & $3.62 \times 10^{-13}$ & $3.56 \times 10^{-13}$ & $3.58 \times 10^{-13}$ & $3.55 \times 10^{-13}$ \\
\hline$S\left(B_{d} \rightarrow J / \psi K_{S}\right)\left(\mathrm{Tot} \approx S M^{\prime}\right)$ & 0.740 & 0.728 & 0.732 & 0.726 \\
\hline
\end{tabular}

Table 1. Predictions of the SUSY SO(10) and G(224) models corresponding to the fit for the fermion massparameters shown in Eq. (20). Either model with the fit as in Eq. (20) leads to the fermion masses and CKM mixings in good agreement with the data (see Eq. (21). The total contribution (denoted by "Tot") represents the sum of 
the $S M^{\prime}$ and the SUSY contributions. Note that the SUSY contribution is important only for $\epsilon_{K}$, furthermore it is relatively negative (as desired) compared to the $S M^{\prime}$ contribution (see discussion in text). The superscript $s . d$. on $\Delta m_{K}$ represents short distance contribution.

In obtaining the entries for the $K$-system we have used central values of the matrix element $\hat{B}_{K}$ and the loop functions $\eta_{i}$ (see Refs. [1,33] for definitions and values) characterizing short distance QCD effects - i.e. $\hat{B}_{K}=0.86 \pm 0.13, f_{K}=159 \mathrm{MeV}, \eta_{1}=1.38 \pm 0.20,{ }^{16} \eta_{2}=$ $0.57 \pm 0.01$ and $\eta_{3}=0.47 \pm 0.04$. For the $B$-system we use the central values of the unquenched lattice results: $f_{B_{d}} \sqrt{\hat{B}_{B_{d}}}=215(11)\left({ }_{-23}^{+0}\right)(15) M e V$ and $f_{B_{s}} \sqrt{\hat{B}_{B_{s}}}=245(10)\left({ }_{-2}^{+3}\right)\left(_{-0}^{+7}\right) \mathrm{MeV}$ [34]. Note that the uncertainties in some of these hadronic parameters are in the range of $15 \%$; thus the predictions of our model as well as that of the SM would be uncertain at present to the same extent.

At this stage the following comments are in order.

(1) In the cases of $\Delta m_{K}, \Delta m_{B_{d}}$ and $S\left(B_{d} \rightarrow J / \psi K_{S}\right)$, the SUSY contributions (with $m_{s q} \sim 0.8-1 \mathrm{TeV}$ and $\left.x \sim 0.5-0.8\right)$ are found to be rather small $(\sim 0.5 \%, 2 \%$, and $3 \%$ respectively) compared to the $S M^{\prime}$ contribution. As a result, for these three entities, the $S M^{\prime}$ contribution practically coincides with the total contribution, which is what is shown in the table. By contrast, for the same spectrum, the SUSY-contribution to $\epsilon_{K}$ is found to be rather sizeable $(\sim 20-25 \%)^{17}$, and importantly enough, negative compared to the $S M^{\prime}$ contribution $^{18}$. The fact that it is relatively negative is an outcome of the model and, as it turns out, is most desirable (see below).

(2) Comparing the predicted values shown in Table 1 with the observed ones (see those listed below Eq. (2) ), together with $\Delta m_{K}^{o b s}=3.47 \times 10^{-15} \mathrm{GeV}$, we see that all four entities including $\epsilon_{K}$ and the asymmetry parameter $S\left(B_{d} \rightarrow J / \psi K_{S}\right)$ agree with the data quite well, for the cases of $\mathrm{SO}(10)$ as well as $\mathrm{G}(224)$ shown in the last two columns (i.e. for $m_{s q} \approx 1$ $\mathrm{TeV}$, and $x \approx 0.8)$, and also for the case of $\mathrm{G}(224)$ in the second column $\left(m_{s q} \approx 800 \mathrm{GeV}\right.$, $x \approx 0.8$ ). In making this comparison we are allowing for plausible (at present theoretically

\footnotetext{
${ }^{16}$ We will be guided by the error of \pm 0.20 on $\eta_{1}$, used in [33], although that quoted in [1] is considerably larger ( \pm 0.53 ).

${ }^{17}$ The fact that the SUSY contribution to $\epsilon_{K}$ (in contrast to those for $\Delta m_{K}, \Delta m_{B_{d}}$ and $S\left(B_{d} \rightarrow J / \psi K_{S}\right)$ ) is relatively large is simply because the SM contribution to $\epsilon_{K}$ is strongly suppressed owing to the smallness of the relevant CKM mixings.

${ }^{18} \mathrm{In}$ as much as we require $\bar{\rho}_{W}^{\prime}$ and $\bar{\eta}_{W}^{\prime}$ to be close to the SM-based phenomenological values (as in Eq. (2)), in accord with the observed values of the fermion masses, CKM-elements and neutrino oscillation parameters, we find that the class of fits satisfying these requirements lead to SUSY-contribution to $\epsilon_{K}$ that is relatively negative compared to the $S M^{\prime}$-contribution.
} 
uncertain but allowed) long distance contribution to $\Delta m_{K}(\sim \pm 15 \%)$, and uncertainties in $\hat{B}_{K}$ or $\eta_{1} \lesssim 10 \%$ (see entries for $\epsilon_{K}$ in the last three columns) and that in $f_{B_{d}} \sqrt{\hat{B}_{B_{d}}}$ by about $3 \%$.

(3) We note that a choice of the SUSY-parameters, e.g. $\left(m_{o}, m_{1 / 2}\right)=(800,250) \mathrm{GeV}$, shown in the table, is in accord with the WMAP-constraint on CMSSM-spectrum in the event that the lightest neutralino is the LSP and represents cold dark matter [35].

(4) It is crucial that the SUSY contribution to $\epsilon_{K}$ (as mentioned above) is significant and is negative relative to the $S M^{\prime}$-contribution. Indeed this is what makes it possible for $\epsilon_{K}(T o t)$ to be desirably lower than the $\epsilon_{K}\left(S M^{\prime}\right)=2.83 \times 10^{-3}$ and thereby to agree better in the last three columns (cases b, c and d) with $\epsilon_{K}^{o b s}=2.27 \times 10^{-3}$. Had the SUSY contribution been positive relative to the $S M^{\prime}$ contribution and still as significant as above, $\epsilon_{K}($ Tot $)$ would have been $(3.34,3.53$, and 3.10$) \times 10^{-3}$ for the cases (b), (c), and (d) respectively, in strong disagreement with observation. In short, the SUSY contribution of the model to $\epsilon_{K}$ has just the right sign and nearly the right magnitude to play the desired role. This seems to be an intriguing feature of the model.

(5) Since the values of the CKM elements including $\bar{\rho}_{W}^{\prime}$ and $\bar{\eta}_{W}^{\prime}$ obtained within our model (see Eq. (21)) are quite close to the SM based phenomenological values (see Ref. [1] and Eq. (2)), we would of course expect that the $S M^{\prime}$ contributions should nearly be the same as the SM contributions, for the same choice of the hadronic parameters $\left(\hat{B}_{K}, \eta_{i}, f_{B_{d}} \sqrt{\hat{B}_{B_{d}}}\right.$ etc.). For instance, using the central values of the parameters given in the recent update of the CKM-triangle analysis by M. Bona et al. [2], that is, $\lambda=\left|V_{u s}\right|=0.2265,\left|V_{c b}\right|=$ $4.14 \times 10^{-2}, \bar{\rho}_{W}=0.172, \bar{\eta}_{W}=0.348, m_{c}=1.3 \mathrm{GeV}$ and $f_{K}=159 \mathrm{MeV}$, and the hadronic parameters as in our case - that is, $\hat{B}_{K}=0.86, \eta_{1}=1.38, \eta_{2}=0.57$, and $\eta_{3}=0.47$ - one obtains $\epsilon_{K}(S M)=2.72 \times 10^{-3}$ which is about $20 \%$ higher than the observed value. Contrast this with the predictions for $\epsilon_{K}$ (Tot) of the $\mathrm{SO}(10)$ or $\mathrm{G}(224)$ models for the cases (b), (c) and (d) in Table 1 where the discrepancies between the predicted and observed value of $\epsilon_{K}$ range from 2 to $12 \%$ with varying signs. At present, such discrepancies, even as high as $20 \%$ for the SM, can of course be accommodated by allowing for uncertainties in $\hat{B}_{K}, \eta_{1}$, and also in $\lambda$.

(6) One main point we wish to stress here, however, is this: At present, the distinctions between the predictions of the SM (in particular for $\epsilon_{K}$ ) versus those of the SUSY SO(10) 
or G(224) models on the one hand, and those between the predictions of the SUSY SO(10) versus the G(224) models on the other hand (compare columns (a), (b), (c) and (d) of Table $1)$ are marred in part because of uncertainties $(\sim 15 \%)$ in the hadronic parameters $\left(\hat{B}_{K}, \eta_{1}\right.$ etc.) as well as that $(\sim 2 \%)$ in $\lambda$, and in part because SUSY is not discovered as yet, and thus the SUSY spectrum is unknown. But once (hopefully) SUSY is discovered at the LHC and thereby the SUSY parameters get fixed, and in addition the uncertainties in the hadronic parameters are reduced (hopefully) to a few percent level through improvements in the lattice calculations, we see from the analysis presented above that we can utilize the combined set of informations to distinguish experimentally between the SM versus the SUSY SO(10)-model versus the SUSY G(224)-model. It is intriguing to see that even low energy experiments involving CP and flavor violations can help distinguish between the SO(10) versus the G(224) models, both of which nearly coincide as regards their predictions for fermion masses and neutrino oscillations. In this way they can shed light on physics at the superheavy scale $M^{*} \gtrsim M_{G U T}$. The experimental distinctions will of course be even sharper once we include predictions for the other processes, some of which are presented below.

(7) $B_{d} \rightarrow \phi K_{S}, \Delta m_{B_{s}}$ and $b \rightarrow s \gamma$ : We now consider the CP violating asymmetry parameter $S\left(B_{d} \rightarrow \phi K_{S}\right)$. For a representative choice of $\left(m_{o}, m_{1 / 2}\right)=(600,300) \mathrm{GeV}$, we get $\delta_{L L}^{23}=(1.40-0.012 i) \times 10^{-2}, \delta_{R R}^{23}=-(5.39+6.27 i) \times 10^{-3}, \delta_{L R}^{23}=-(0.29+3.08 i) \times 10^{-4} / \tan \beta$ and $\delta_{R L}^{23}=-(1.92+2.70 i) \times 10^{-4} / \tan \beta$ as predictions of our model (see Eqs. (13) and (19)) ). It is easy to verify that the SUSY-amplitude for this decay in our model is only of order $1 \%$ (or less) [36] compared to that in the SM. As a result, adding $S M^{\prime}$ and SUSY contributions to the decay amplitudes, we obtain:

$$
S\left(B_{d} \rightarrow \phi K_{S}\right)\left(T o t \approx S M^{\prime}\right) \approx 0.728
$$

Allowing for variant fits which also give fermion masses and CKM mixings in good agreement with observations, we find that $S\left(B_{d} \rightarrow \phi K_{S}\right)$ should lie in the range of $\approx+0.65$ to +0.73 . Thus our model predicts that $S\left(B_{d} \rightarrow \phi K_{S}\right)$ is close to the $S M$ prediction $(\approx 0.70 \pm 0.10)$ and certainly not negative in sign. ${ }^{19}$ When we started writing this paper, BaBar and BELLE data were yielding widely differing values of $(0.45 \pm 0.43 \pm 0.07)$ and $\left(-0.96 \pm 0.50_{-0.07}^{+0.09}\right)$ respectively for $S\left(B_{d} \rightarrow \phi K_{S}\right)[37]$. Most recently, the two groups reported

\footnotetext{
${ }^{19}$ Our prediction in this regard was reported at the Fujihara seminar [7], held February 23-25, 2004.
} 
new values for the asymmetry parameter $S\left(B_{d} \rightarrow \phi K_{S}\right)=\left(+0.50 \pm 0.25_{-0.04}^{+0.07}\right)_{\text {BaBar }} ;(+0.06 \pm$ $0.33 \pm 0.09)_{B E L L E}[37]$, at the Beijing International Conference on High Energy Physics . Meanwhile there have been many theoretical and phenomenological attempts $[36,38]$ to obtain possible large deviations in $S\left(B_{d} \rightarrow \phi K_{S}\right)$ from the SM-value, including, in some cases, negative values for the same (as suggested by the earlier BELLE data). It will thus be extremely interesting from the viewpoint of the $\mathrm{G}(224) / \mathrm{SO}(10)$-framework presented here to see whether the true value of $S\left(B_{d} \rightarrow \phi K_{S}\right)$ will turn out to be close to the SM-prediction or not.

Including contributions from $\delta_{L L, R R}^{23}$ and $\delta_{L R, R L}^{23}$ (as predicted in our model), we get:

$$
\Delta m_{B_{s}}\left(T o t \approx S M^{\prime}\right) \approx 17.3 p s^{-1}\left(\frac{f_{B_{s}} \sqrt{\hat{B}_{B_{s}}}}{245 M e V}\right)^{2} .
$$

This is of course compatible with the present lower limit on $\Delta m_{B_{s}} \gtrsim 14.4 p s^{-1}[2]$.

Using $\delta_{R L}^{23}$ given above, we obtain $A\left(b_{L} \rightarrow s_{R} \gamma\right)_{\tilde{g}} \approx(1-1.5) \times 10^{-10} \mathrm{GeV}^{-1} / \tan \beta$. Even allowing for variant fits, the SUSY-amplitude, in this case, is found to be only about (1.5$5) \%$ of the SM amplitude. The same conclusion holds also for $A\left(b_{R} \rightarrow s_{L} \gamma\right)_{\tilde{g}}$. In short, our results for $\left(B_{d} \rightarrow \phi K_{S}\right), \Delta m_{B_{s}}$ and $b \rightarrow s \gamma$ nearly coincide with those of the SM.

(8) Contribution of the A term to $\epsilon_{K}^{\prime}$ : Direct $\mathrm{CP}$ violation in $K_{L} \rightarrow \pi \pi$ receives a new contribution from the chromomagnetic operator $Q_{g}^{-}=\left(g / 16 \pi^{2}\right)\left(\bar{s}_{L} \sigma^{\mu \nu} t^{a} d_{R}-\bar{s}_{R} \sigma^{\mu \nu} t^{a} d_{L}\right) G_{\mu \nu}^{a}$, which is induced by the gluino penguin diagram. This contribution is proportional to $X_{21} \equiv$ $\operatorname{Im}\left[\left(\delta_{L R}^{d}\right)_{21}-\left(\delta_{L R}^{d}\right)_{12}^{*}\right]$, which is predictable in our model (see Eqs. (18) and (19)). Following Refs. [39] and [40], one obtains:

$$
R e\left(\epsilon^{\prime} / \epsilon\right)_{\tilde{g}} \approx 91 B_{G}\left(\frac{110 \mathrm{MeV}}{m_{s}+m_{d}}\right)\left(\frac{500 \mathrm{GeV}}{m_{\tilde{g}}}\right) X_{21}
$$

where $B_{G}$ is the relevant hadronic matrix element. Model-dependent considerations (allowing for $m_{K}^{2} / m_{\pi}^{2}$ corrections) indicate that $B_{G} \approx 1-4$, and that it is positive [39]. Using the prediction of our model (via Eqs. (18) and (19)), for a typical SUSY- spectrum used in previous considerations (e.g. $\left.\left(m_{o}, m_{1 / 2}\right)=(600,300) G e V\right)$, we obtain: $X_{21} \approx 2.1 \times$ $10^{-5} / \tan \beta$. Note that the sign of $X_{21}$, as derived in the model, is positive. Inserting this in Eq. (24), and putting $\left(m_{s}+m_{d}\right) \approx 110 \mathrm{MeV}$, we get:

$$
\operatorname{Re}\left(\epsilon^{\prime} / \epsilon\right)_{\tilde{g}} \approx+\left(8.8 \times 10^{-4}\right)\left(B_{G} / 4\right)(5 / \tan \beta) .
$$


We see that if the positive sign of $B_{G}$ is confirmed by reliable lattice calculations, the gluino penguin contribution in our model can quite plausibly give a significant positive contribution to $\operatorname{Re}\left(\epsilon^{\prime} / \epsilon\right)_{\tilde{g}} \approx(4-14) \times 10^{-4}$, depending upon $B_{G} \approx 2-4$ and $\tan \beta \approx(3-10)$. At present the status of SM contribution to $\operatorname{Re}\left(\epsilon^{\prime} / \epsilon\right)$ is rather uncertain. For instance, the results of Ref. [41] and [42] based on quenched lattice calculations in the lowest order chiral perturbation theory suggest negative central values for $\operatorname{Re}\left(\epsilon^{\prime} / \epsilon\right)$. (To be specific Ref. [41] yields $\operatorname{Re}\left(\epsilon^{\prime} / \epsilon\right)_{S M}=$ $(-4.0 \pm 2.3) \times 10^{-4}$, the errors being statistical only.) On the other hand, using methods of partial quenching [43] and staggered fermions, positive values of $\operatorname{Re}\left(\epsilon^{\prime} / \epsilon\right)$ in the range of about $(3-13) \times 10^{-4}$ are obtained in [44]. In addition, a recent non-lattice calculation based on next-to-leading order chiral perturbation theory yields $R e\left(\epsilon^{\prime} / \epsilon\right)_{S M}=\left(19 \pm 2_{-6}^{+9} \pm 6\right) \times 10^{-4}$ [45]. The systematic errors in these calculations are at present hard to estimate. The point we wish to note here is that the SUSY-contribution to $\operatorname{Re}\left(\epsilon^{\prime} / \epsilon\right)$, in our model, is significant, and when the dust settles, following a reliable calculation of $\operatorname{Re}\left(\epsilon^{\prime} / \epsilon\right)$ in the SM, it would be extremely interesting to check whether the SUSY-contribution obtained here is playing an important role in accounting for the observed value given by $\operatorname{Re}\left(\epsilon^{\prime} / \epsilon\right)_{o b s}=(17 \pm 2) \times 10^{-4}[46]$ or not.

(9) EDM of the neutron and the electron: RG-induced $A$-terms of the model generate chirality-flipping sfermion mixing terms $\left(\delta_{L R}^{d, u, l}\right)_{i j}$, whose magnitudes and phases are predictable in the model (see Eqs. (18) and (19)), for a given choice of the universal SUSYparameters $\left(m_{o}, m_{1 / 2}\right.$, and $\left.\tan \beta\right)$. These contribute to the EDM's of the quarks and the electron by utilizing dominantly the gluino and the neutralino loops respectively. We will approximate the latter by using the bino-loop. These contributions are given by (see e.g. [47]):

$$
\begin{gathered}
\left(d_{d}, d_{u}\right)_{A_{i n d}}=\left(-\frac{2}{9}, \frac{4}{9}\right) \frac{\alpha_{s}}{\pi} \text { e } \frac{m_{\tilde{g}}}{m_{s q}^{2}} f\left(\frac{m_{\tilde{g}}^{2}}{m_{s q}^{2}}\right) \operatorname{Im}\left(\delta_{L R}^{d, u}\right)_{11} \\
\left(d_{e}\right)_{A_{i n d}}=-\frac{1}{4 \pi} \frac{\alpha_{e m}}{\cos ^{2} \theta_{W}} \text { e } \frac{m_{\tilde{B}}}{m_{l}^{2}} f\left(\frac{m_{\bar{B}}^{\tilde{B}}}{m_{\bar{l}}^{2}}\right) \operatorname{Im}\left(\delta_{L R}^{l}\right)_{11} .
\end{gathered}
$$

For a representative choice $\left(m_{o}, m_{1 / 2}\right)=(600,300) \mathrm{GeV}$ (i.e. $m_{s q}=1 \mathrm{TeV}, m_{\tilde{g}}=900 \mathrm{GeV}$, $m_{\tilde{l}}=636 \mathrm{GeV}$ and $m_{\tilde{B}}=120 \mathrm{GeV}$ ), using Eqs. (18) and (19), we get:

$\left(d_{d}\right)_{A_{i n d}}=\frac{4.15 \times 10^{-26}}{\tan \beta} \mathrm{ecm} ; \quad\left(d_{u}\right)_{A_{\text {ind }}}=\left(-1.6 \times 10^{-26}\right) \mathrm{ecm} ; \quad\left(d_{e}\right)_{A_{i n d}}=\frac{1.1 \times 10^{-28}}{\tan \beta} \mathrm{ecm}$

The EDM of the neutron is given by $d_{n}=\frac{1}{3}\left(4 d_{d}-d_{u}\right)$. Thus for $\mathrm{SO}(10)$, with the choice of $\left(m_{o}, m_{1 / 2}\right)$ as above, we get

$$
\left(d_{n}\right)_{A_{\text {ind }}}=(1.6,1.08) \times 10^{-26} \text { ecm for } \tan \beta=(5,10) .
$$


Note that these induced $A$-term contributions are larger for smaller $\tan \beta$. For an alternative choice $\left(m_{o}, m_{1 / 2}\right)=(800,250) \mathrm{GeV}$, which as mentioned before is compatible with the WMAP/CDM-constraint [35], the predicted EDM of the neutron and the electron are reduced respectively by about $36 \%$ and a factor of 3.6. The predictions for the $\mathrm{G}(224)$-model are smaller than those for the $\mathrm{SO}(10)$-model by nearly a factor of two in all cases.

We should also note that intrinsic SUSY phases denoted by $\phi_{A}=\arg \left(A_{o}^{*} m_{1 / 2}\right)$ and $\phi_{B}=\arg \left(m_{1 / 2} \mu B^{*}\right)$, if present, would make additional contributions to EDM's through gluino and/or chargino/neutralino loops, which should be added to the contributions shown above. These contributions have been widely discussed in the literature (see e.g. [47]). As is well known, with $A_{o}=0$ or small $(\lesssim 1 \mathrm{GeV})$ at the scale $M^{*}$ (as we have chosen, following the examples of Refs. [19] and [21]), these contributions are proportional to $\left(m_{d, e}\right) \mu \tan \beta\left(\sin \phi_{B}\right)$. They would be typically about 50-300 times larger than the values shown above (Eqs. (27) and (28)), if the relevant intrinsic SUSY phases are nearly unity. This is the familiar SUSY $\mathrm{CP}$ problem. The point of the present paper is that even if the intrinsic SUSY phases are naturally zero or insignificantly small, as would be true in a theory where the SUSY CP problem is naturally solved ${ }^{20,21}$, the induced A-term contributions to EDM's (arising from GUT-scale physics) as presented above would still be present. The interesting point is that these contributions are completely determined in magnitude and phase within our model (for a given choice of the SUSY universal parameters $\left.\left(m_{o}, m_{1 / 2}, \tan \beta\right)\right)$.

Given the experimental limits $d_{n}<6.3 \times 10^{-26}$ e cm [51] and $d_{e}<4.3 \times 10^{-27}$ e cm [52], we

\footnotetext{
${ }^{20} \mathrm{~A}$ possible solution to the SUSY CP problem could arise as follows. Assume that CP violation arises spontaneously, only in the visible sector, through the VEV's of fields at the GUT-scale, like those of $\mathbf{1 6}_{H}, \overline{\mathbf{1 6}}_{H}, \mathbf{4 5}_{H}$ and the singlet $S$. One can argue that the VEV's of at least some of these can be naturally complex or purely imaginary, consistent with the minimization of the potential, even if all parameters in the potential are real. In this case, intrinsic SUSY phases like those in $m_{1 / 2}, A$ and $B$ are, of course, zero. Now if the $\mu$-term can be derived, through a satisfactory resolution of the $\mu$-problem, for example, either by the Giudice-Masiero mechanism [48], or by the ideas suggested in [49], or by involving a coupling in the superpotential of the form [50]: $\kappa \mathbf{1 0}_{H} \mathbf{1 0}_{H} N+\lambda N^{3}+\ldots$, where the singlet $N$ is not allowed to couple to the other fields mentioned above, and acquires a real VEV of order $1 \mathrm{TeV}$ (as needed), with $\kappa$ and $\lambda$ being real, then the $\mu$-term would also be real. In this case, all intrinsic SUSY phases would disappear. We plan to explore this possibility in a future work.

${ }^{21}$ An alternative resolution of the SUSY CP problem arises in a class of gaugino mediated SUSY-breaking (with the $\mu$ problem being resolved for example as in [48]) in which all relevant SUSY parameters become proportional to $m_{1 / 2}$ [19]. A third resolution of the SUSY CP problem would arise in a model where both $A$ and $B$ terms are naturally zero or sufficiently small at the scale $M^{*}$. This is precisely what happens in, for example, the anomalous $U(1)_{A}$ model of SUSY-breaking that arises in the context of a three-family string-solution [21]. In this case, extra gauge symmetries of the model suppress both $A$ and $B$ terms at $M^{*}$.
} 
see that the predictions of the model (arising only from the induced $A$-term contributions) especially for the EDM of the neutron is in an extremely interesting range suggesting that it should be discovered with an improvement of the current limit by a factor of about 10 .

\section{Summary and Concluding Remarks}

In this paper we have explored the possibility that (a) fermion masses, (b) neutrino oscillations, (c) CP-non conservation and (d) flavor violations get intimately linked to each other within supersymmetric grand unification, especially that based on the symmetry $\mathrm{SO}(10)$ or an effective symmetry $\mathrm{G}(224)=S U(2)_{L} \times S U(2)_{R} \times S U(4)^{c}$. In this context, we extend the framework proposed previously in [9], which successfully described fermion masses and neutrino oscillations (see Sec. 2), to include CP violation. We assume, in the interest of predictiveness, that CP-violation, arising through the SM as well as SUSY interactions, has its origin entirely (or primarily) through phases in the fermion mass matrices. We also assume that flavor-blind universal SUSY parameters $\left(m_{o}, m_{1 / 2}\right.$ and $\tan \beta$ with $A_{o}$ being small or real) characterize SUSY-breaking effects at a high scale $M^{*} \gtrsim M_{G U T}$. In this case, all the weak scale CP and/or flavor-violating as well as flavor-preserving sfermion transition-elements $\delta_{L L, R R, L R}^{i j}$, and the induced A-parameters, get fully determined within the model, in their magnitudes as well as in phases, simply by the entries in the $\mathrm{SO}(10)$-based fermion massmatrices, once the few soft parameters $\left(m_{o}, m_{1 / 2}\right.$ and $\left.\tan \beta\right)$ are specified. This is how CP and flavor violations arising jointly from the SM and SUSY interactions, get intimately tied to fermion masses and neutrino oscillations, within a predictive $\mathrm{SO}(10) / \mathrm{G}(224)$-framework outlined above. The presence of GUT-scale physics induces enhanced flavor violation with and without $\mathrm{CP}$ violation, which provides a distinguishing feature of the model ${ }^{22}$, relative to CMSSM or MSSM. ${ }^{23}$

\footnotetext{
${ }^{22}$ Even in the case of CMSSM, all the parameters of MSSM at the electroweak scale (some 105 of them) are of course also all fully determined in terms of the SUSY-parameters $\left(m_{o}, m_{1 / 2}\right.$ and $\left.\tan \beta\right)$ and those involving the fermion masses and mixings. However, in this case, as mentioned in Sec. 4, owing to the absence of GUT-scale physics in the interval $M^{*} \rightarrow M_{G U T}$, the most interesting effects on the entities considered here (e.g. those on $\epsilon_{K}, \epsilon_{K}^{\prime}$ and the EDM's) would be absent or negligibly small.

${ }^{23}$ While we have focussed in this paper on the $\mathrm{SO}(10) / \mathrm{G}(224)$-model of Ref. [9], we note that generically such enhanced flavor and/or CP violations arising from GUT-scale physics would of course be present in alternative models of SUSY grand unification [53] as well, as long as the messenger scale $M^{*}$ lies above $M_{G U T}$. The detailed predictions and consistency of any such model as regards flavor and/or CP violations can however depend (even sensitively) upon the model, and this is worth
} 
As mentioned in Secs. 1 and 5, the framework presented above faces, however, a primafacie challenge. Including SM and SUSY contributions, the question arises, can the framework successfully describe the observed features of CP and flavor-violations including those listed in Eq. (11), while retaining the successes of the CP-preserving framework [9] as regards fermion masses and neutrino oscillations? Our work here shows that the SUSY $\mathrm{SO}(10) / \mathrm{G}(224)$-framework proposed here, which extends the framework of Ref. [9], indeed meets this challenge squarely. In the process, it makes several predictions, only some of which are considered here; these can eventually help distinguish the framework from other alternatives.

Our results can be summarized as follows:

(1) It is found that, with allowance for phases, there exists a fit to the parameters of the fermion mass-matrices (Eq. (3) ) which successfully describes fermion masses, all the CKM elements and neutrino oscillations as in Ref. [9] (see Eq. (21)), and simultaneously yields the Wolfenstein parameters $\left(\bar{\rho}_{W}^{\prime}, \bar{\eta}_{W}^{\prime}\right)$ that are close to the phenomenological SM values (Eq. (2) ). The merit of obtaining such values for $\left(\bar{\rho}_{W}^{\prime}, \bar{\eta}_{W}^{\prime}\right)$ in accounting for the data on CP and flavor violations in quantities such as those listed in Eq. (11) has been stressed in Sec. 5.

(2) With these values of $\left(\bar{\rho}_{W}^{\prime}, \bar{\eta}_{W}^{\prime}\right)$, and a plausible choice of the SUSY-spectrum ${ }^{24}$ (i.e. $m_{s q} \approx(600-1000) \mathrm{GeV}$ and $m_{\tilde{g}} \approx(500-900) \mathrm{GeV}$, say, it is found that the derived values of all four quantities (i.e. $\Delta m_{K}, \epsilon_{K}, \Delta m_{B_{d}}$ and $S\left(B_{d} \rightarrow J / \psi K_{S}\right)$ agree with the data quite well (allowing for up to $15 \%$ uncertainty in hadronic matrix elements), see Table 1.

(3) Although the $S M^{\prime}$ contributions (for the fit shown in Eq. (201) ) nearly coincide with the SM contributions to all entities, and SUSY-contributions to entities such as $\Delta m_{K}, \Delta m_{B_{d}}$ and $S\left(B_{d} \rightarrow J / \psi K_{S}\right)$ are rather small $(\lesssim$ a few $\%)$, the contributions from SUSY, as a rule, are nevertheless prominent (of order 20-25\%) especially when the SM (or $S M^{\prime}$ ) contributions are suppressed (for example due to smallness of the mixing angles). Such is precisely the case for $\epsilon_{K}, \epsilon_{K}^{\prime}$ and the edm's of the neutron and the electron, (as well as for lepton flavor violating processes to be discussed in a forthcoming paper [25]). It is found that the SUSYcontribution to $\epsilon_{K}$ is sizable (of order 20-25\%) and negative relative to the $S M^{\prime}$ contribution, just as desired, to yield better agreement between the predicted and the observed value (see examining.

${ }^{24}$ Lighter masses for the SUSY particles like $m_{s q} \approx 600 \mathrm{GeV}$ and $m_{\tilde{g}} \approx 500 \mathrm{GeV}$ (say) are allowed for the case of G(224), though not for $\mathrm{SO}(10)$ (see discussion following Table 1). 
Table 1).

(4) The sizable negative contribution of SUSY to $\epsilon_{K}$ in our model provides an important tool to help distinguish not only between the SM versus the SUSY SO(10)/G(224)-models, but also between the $\mathrm{SO}(10)$ and the $\mathrm{G}(224)$-models themselves (see Table 1). Such distinctions would be possible once (hopefully) SUSY is discovered at the LHC and thereby the SUSY parameters get fixed, and in addition the uncertainties in the hadronic parameters $\left(\hat{B}_{K}\right.$ and $\eta_{1}$ ) are reduced to (say) a $5 \%$ level or better, through improved lattice calculations.

(5) The model predicts that $\mathrm{S}\left(B_{d} \rightarrow \phi K_{S}\right)$ should lie in the range of $+(0.65-0.73)$, i.e. close to the SM-prediction. Given the present still significant disparities between the BaBar and BELLE results versus the SM-predictions, it would be interesting to see where the true value of $\mathrm{S}\left(B_{d} \rightarrow \phi K_{S}\right)$ would turn out to lie.

(6) It is interesting that the quantity $X_{21}=\operatorname{Im}\left[\left(\delta_{L R}^{d}\right)_{21}-\left(\delta_{L R}^{d}\right)_{12}^{*}\right]$, relevant for $\epsilon_{K}^{\prime}$, is found to be positive in the model. If the presently indicated sign of the relevant hadronic matrix element $B_{G}$ being positive is confirmed, our model would give a positive contribution to $\operatorname{Re}\left(\epsilon^{\prime} / \epsilon\right)$ which quite plausibly can lie in the range of $+(4-14) \times 10^{-4}$. While this is in the interesting range, its relevance can be assessed only after the associated matrix elements are determined reliably.

(7) The model predicts that the EDM's of the neutron and the electron should be discovered with improvements in the current limits by factors of 10 and 100 respectively. (Intrinsic SUSY-phases, even if present, would not alter this conclusion as long as there is no large cancellation between different contributions.)

(8) It would be most interesting to explore the consequences of the model, involving SUSY contributions, to other processes such as $B_{s} \rightarrow J / \psi \phi, B_{s} \rightarrow \phi K_{S}, B \rightarrow K \pi, B \rightarrow \pi \pi$, $B \rightarrow D K, K_{L} \rightarrow \pi \nu \bar{\nu}, K^{+} \rightarrow \pi \nu \bar{\nu}$, and especially lepton violating processes (such as $\mu \rightarrow e \gamma, \tau \rightarrow \mu \gamma, \tau \rightarrow e \gamma$ etc.). We stress that the net (SM' + SUSY)-contributions to all these processes involving $\mathrm{CP}$ and/or flavor violations are completely determined within our model. They do not involve any new parameters. For this reason, the model turns out to be highly predictive and thoroughly testable. These processes are under study; a paper on lepton flavor violation is in preparation.

To conclude, the SUSY SO(10)/G(224) framework, as proposed in Ref. [9] and extended here, subject to the assumption of universality of SUSY parameters, drastically reduces the 
parameters for SUSY-contributions to CP and flavor-violations. In effect, the extension proposed here ties in fermion masses, neutrino oscillations, $\mathrm{CP}$ and flavor violations within a predictive and testable framework.

\section{Acknowledgements}

We would like to thank Antonio Masiero, Rabindra Mohapatra, Qaisar Shafi and Amarjit Soni for helpful discussions and Abolhassan Jawahery for a helpful correspondence. The work of KSB is supported in part by the Department of Energy Grant Nos. DE-FG0204ER46140, DE-FG02-04ER41306 and by an award from Research Corporation. That of JCP is supported in part by Department of Energy Grant No. DE-FG-02-96ER-41015.

\section{References}

[1] See e.g. M. Ciuchini, E. Franco, F. Parodi, V. Lubicz, L. Silvestrini, and A. Stocchi, Talk at "Workshop on the CKM Unitarity Triangle", Durham, April 2003, hep-ph/0307195.

[2] An extensive analysis appears in the Proceedings of "The CKM Matrix and the Unitarity Triangle", ed. by M. Battaglia, A. J. Buras, P. Gambino and A. Strochhi, hep-ph/0304132, For a very recent update, see M. Bona et al., hep-ph/0408079.

[3] B. Aubert et al. (BaBar Collaboration), Published in ICHEP 2002, Amsterdam 2002, 481-484, hep-ex/0207042 K. Abe et al. (BELLE Collaboration), Phys. Rev. D66 071102 (2002), hep-ex/0208025.

[4] M. Fukugita and T. Yanagida Phys. Lett. B174, 45 (1986); G. Lazarides and Q. Shafi, Phys. Lett, B258, 305 (1991); M.A. Luty, Phys. Rev. D45, 455 (1992). In the context of the model to be presented here, see J.C. Pati, Phys. Rev. D68, 072002 (2003), hep-ph/0209160.

[5] J.C. Pati and A. Salam, Phys. Rev. Lett. 31, 661 (1973); Phys. Rev. D10, 275 (1974).

[6] H. Georgi, in Particles and Fields, Ed. by C. Carlson (AIP, NY, 1975), p.575; H. Fritzsch and P. Minkowski, Ann. Phys. 93, 193 (1975). 
[7] J.C. Pati, "Neutrino Masses: Shedding light on Unification and Our Origin", Talk given at the Fujihara Seminar, KEK Laboratory, Tsukuba, Japan, February 23-25, 2004, hep-ph/0407220, to appear in the proceedings.

[8] P. Minkowski, Phys. Lett. B67, 421 (1977); M. Gell-Mann, P. Ramond and R. Slansky, in: Supergravity, eds. F. van Nieuwenhuizen and D. Freedman (Amsterdam, North Holland, 1979) p. 315; T. Yanagida, in: Workshop on the Unified Theory and Baryon Number in the Universe, eds. O. Sawada and A. Sugamoto (KEK, Tsukuba) 95 (1979); S.L. Glashow, in Quarks and Leptons, Cargese 1979, eds. M. Levy et al. (Plenum 1980) p. 707; R. N. Mohapatra and G. Senjanovic, Phys. Rev. Lett. 44, 912 (1980).

[9] K. S. Babu, J. C. Pati and F. Wilczek, "Fermion masses, neutrino oscillations, and proton decay in the light of SuperKamiokande" hep-ph/9812538, Nucl. Phys. B566, 33 (2000).

[10] These have been introduced in various forms in the literature. For a sample, see e.g., C. D. Frogatt and H. B. Nielsen, Nucl. Phys. B147, 277 (1979); L. Hall and H. Murayama, Phys. Rev. Lett. 75, 3985 (1995); P. Binetruy, S. Lavignac and P. Ramond, Nucl. Phys. B477, 353 (1996). In the string theory context, see e.g., A. Faraggi, Phys. Lett. B278, 131 (1992).

[11] The zeros in "11", "13" and " 31 " elements signify that they are relatively small quantities (specified below). While the "22" elements were set to zero in Ref. [9], because they are meant to be <"23" "32"/ "33" 10 $0^{-2}$ (see below), and thus unimportant for purposes of Ref. [9], they are retained here, because such small $\zeta_{22}^{u}$ and $\zeta_{22}^{d}\left[\sim(1 / 3) \times 10^{-2}\right.$ (say)] can still be important for CP violation and leptogenesis.

[12] For $\mathrm{G}(224)$, one can choose the corresponding sub-multiplets - that is, $(1,1,15)_{H},(1$, $2, \overline{4})_{H},(1,2,4)_{H},(2,2,1)_{H}$ - together with a singlet $S$, and write a superpotential analogous to Eq. (44).

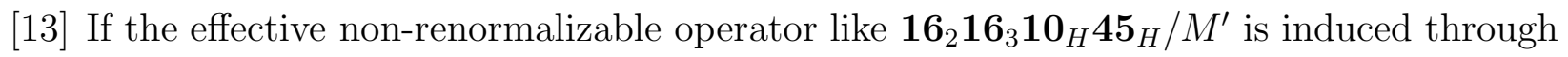
exchange of states with GUT-scale masses involving renormalizable couplings, rather than through quantum gravity, $M^{\prime}$ would, however, be of order GUT-scale. In this case $\left\langle\mathbf{4 5} \mathbf{5}_{H}\right\rangle / M^{\prime} \sim 1$, rather than $1 / 10$. 
[14] While $\mathbf{1 6}_{H}$ has a GUT-scale VEV along the SM singlet, it turns out that it can also have a VEV of EW scale along the " $\tilde{\nu}_{L}$ " direction due to its mixing with $\mathbf{1 0}_{H}^{d}$, so that the $H_{d}$ of MSSM is a mixture of $\mathbf{1 0}_{H}^{d}$ and $\mathbf{1 6}_{H}^{d}$. This turns out to be the origin of non-trivial CKM mixings (see Ref. [9]).

[15] The flavor charge(s) of $\mathbf{4} \mathbf{5}_{H}\left(\mathbf{1 6}_{H}\right)$ would get determined depending upon whether $p(q)$ is one or zero (see below).

[16] J. C. Pati, "Probing Grand Unification Through Neutrino Oscillations, Leptogenesis and Proton Decay", hep-ph/0305221, Proceedings of Erice Summer School, 2002, p.194-236, Ed. by A. Zichichi, Publ. World Scientific.

[17] L. Wolfenstein, Phys. Rev. Lett. 51, 1945 (1983).

[18] A. H. Chamseddine, R. Arnowitt and P. Nath, Phys. Rev. Lett. 49, 970 (1982); R. Barbieri, S. Ferrara and C. A. Savoy, Phys. Lett. B119, 343 (1982); L. J. Hall, J. Lykken and S. Weinberg, Phys. Rev. D27, 2359 (1983); L. Alvarez-Gaume, J. Polchinski and M. B. Wise, Nucl. Phys. B221, 495 (1983).

[19] Z. Chacko, M. A. Luty, A. E. Nelson and E. Ponton, JHEP, 0001, 003 (2000); D. E. Kaplan, G. D. Kribs amd M. Schmaltz, Phys. Rev. D62, 035010 (2000).

[20] G. Dvali and A. Pomarol, Phys. Rev. Lett. 77, 3728 (1996); P. Binetruy and E. Dudas, Phys. Lett. B389, 503 (1996).

[21] A. Faraggi and J.C. Pati, Nucl. Phys. B256, 526 (1998); hep-ph/9712516v3.

[22] N. Arkani-Hamed, M. Dine and S. P. Martin, hep-ph/9803432, Phys. Lett. B431, 329 (1998).

[23] R. Barbieri, L. J. Hall and A. Strumia, Nucl. Phys. B445, 219 (1995); hep-ph/9501334. For analogous considerations see also L. J. Hall, V. A. Kostelecky and S. Raby, Nucl. Phys B267, 415 (1986); F. Borzumati and A. Masiero, Phys. Rev. Lett. 57, 961 (1986).

[24] M. Carena, M. Olechowski, S. Pokorski, and C. E. M. Wagner, Nucl. Phys. B419, 213 (1994); hep-ph/9311222 
[25] K. S. Babu, J. C. Pati and P. Rastogi, "Linking Lepton Flavor Violation with Fermion masses, Neutrino Oscillations and CP Violation in SO(10)/G(224)" , to appear.

[26] H. S. Goh, R. N. Mohapatra and Siew-Phang Ng, Phys. Rev. D68 115008 (2003), hep-ph/0308197.

[27] For a partial list of refereces for works along these lines see e.g. H. Arason et al. Phys. Rev. D 46, 3945 (1992); D. J. Castano, E. J. Pirad, P. Ramond, Phys. Rev. D49, 4882 (1994); K. S. Babu, C. N. Leung and J. Pantaleone, Phys. Lett. B319, 191 (1993); H. Fusaoka, Y. Koide, Phys. Rev. D57, 3986 (1998); C. R. Das, M. K. Parida, Eur. Phys. J. C20, 121 (2001).

[28] For very recent improved lattice results on light quark masses, see C. Aubin et al. (HPQCD, MILC and UKQCD collaboration), Phys. Rev. D70 031504 (2004), hep-lat/0405022, and C. Aubin et al. (MILC collaboration), hep-lat/0407028. These papers give: $m_{s}^{\overline{M S}}(2 \mathrm{GeV})=76(0)(3)(7)(0) \mathrm{MeV}, 1 / 2\left(m_{u}+m_{d}\right)^{\overline{M S}}(2 \mathrm{GeV})=$ $2.8(0)(1)(3)(0) \mathrm{MeV}$ and $m_{u} / m_{d}=0.43(0)(1)(8)$. Using these results and extrapolating to $1 \mathrm{GeV}$, one gets: $m_{u}(1 \mathrm{GeV}) \approx 2.1 \pm 0.5 \mathrm{MeV}, m_{d}(1 \mathrm{GeV}) \approx 4.9 \pm 0.6 \mathrm{MeV}$ and $m_{s}(1 \mathrm{GeV}) \approx 95 \pm 10 \mathrm{MeV}$. Note that these values are considerably smaller than those commonly used in literature. Somewhat larger values of the masses are quoted in a paper, also based on improved lattice-calculation, by M. Goeckeler et al. (QCDSFUKQCD Collaboration) hep-ph/0409312, which appeared on the web just before the completion of this paper.

[29] For CKM elements, see e.g. Ref. [1] or most recent update by M. Bona et al. hep-ph/0408079 in Ref. [2]. The bottom quark mass quoted in C. W. Bauer et al. (Phys. Rev. D67, 054012 (2003)) is: $m_{b}\left(m_{b}\right)=4.22 \pm 0.09 \mathrm{GeV}$, which is lower than our value given in Eq.(21) by about 10-12\%. Gluino-loop corrections to $m_{b}$ for $\mu<0$ and $\tan \beta=10$ (say) can, however, reduce $m_{b}$ by about $6-7 \%$.

[30] F. Gabbiani, E. Gabrielli, A. Masiero, L. Silvestrini, Nucl. Phys. B477, 321 (1996); hep-ph/9604387.

[31] M. Ciuchini et al., JHEP 9810:008 (1998), hep-ph/9808328. 
[32] S. Khalil and E. Kou, Phys. Rev. D67, 055009 (2003); hep-ph/0212023.

[33] A. J. Buras, Proceedings of the International School of Subnuclear Physics, Erice, Italy 2000, p 200-337, edited by A. Zichichi, Publ. by World Scientific; hep-ph/0101336.

[34] S. Aoki et al., JLQCD Collaboration, Phys. Rev. Lett. 91, 212001 (2003), hep-ph/0307039.

[35] For a recent analysis, that allows for uncertainties in $\left(m_{t}, m_{b}\right)$, and for the relevant references, see e.g. J. Ellis, K. Olive, Y. Santoso and V. Spanos, Phys. Rev. D69, 095004 (2004), hep-ph/0310356.

[36] E. Lunghi, D. Wyler, Phys. Lett. B521 320 (2001), hep-ph/0109149 S. Khalil and E. Kou, Phys. Rev. D67, 055009 (2003); hep-ph/0212023 R. Harnik, D. T. Larson, H. Murayama, A. Pierce, Phys. Rev. D69,094024 (2004), hep-ph/0212180.

[37] B. Aubert et al. (BaBar Collaboration), hep-ex/0403026 K. Abe et al. (BELLE Collaboration), Phys. Rev. Lett. 91, 261602 (2003); The most recent results on $S\left(B_{d} \rightarrow \phi K_{S}\right)$, submitted to the $32^{\text {nd }}$ International Conference of High Energy Physics (Aug. 16-22, 2004), Beijing, China, appear in the papers of B. Aubert et al. (BaBar Collaboration), hep-ex/0408072, and K. Abe et al. (BELLE Collaboration), hep-ex/0409049.

[38] See e.g. D. Chang, A. Masiero and H. Murayama, Phys. Rev. D67, 075013 (2003), hep-ph/0205111; T. Moroi, Phys. Lett. B493, 366 (2000), hep-ph/0007328; G.L. Kane, P. Ko, H. Wang, C. Kolda, J. Park, L. Wang, Phys. Rev. D70, 035015 (2004), hep-ph/0212092 J. Park, hep-ph/0312118 J. Hisano and Y. Shimizu, Phys. Lett. B565, 183 (2003), hep-ph/0303071; T. Goto, Y. Okada, Y. Shimizu, T. Shindou and M. Tanaka, Phys. Rev. D70, 0305012 (2004), hep-ph/0306093.

[39] A. J. Buras, G. Colangelo, G. Isidori, A. Romanino, and L. Silvestrini, Nucl. Phys. B566,3 (2000); hep-ph/9908371.

[40] Y. Nir, Lectures Given at Scottish Univ. Summer School, Scotland 2001; hep-ph/0109090. 
[41] T. Blum et al. (RBC collab.), Phys. Rev. D68, 114506 (2003), hep-lat/0110075. See A. Soni, Talk at Pascos 2003 Conference, Mumbai (India), Pramana 62415 (2004), hep-ph/0307107, for a critical review of this and similar works.

[42] J. Noaki et al., Phys. Rev. D68, 014501 (2003).

[43] M. Golterman and E. Pallante, JHEP 0110, 037 (2001), Phys. Rev. D69, 074503 (2004).

[44] T. Bhattacharya, G. T. Fleming, R. Gupta, G. Kilcup, W. Lee and S. Sharpe, hep-lat/0409046.

[45] A. Pich, hep-ph/0410215 E. Pallante, A. Pich and I. Scimemi, Nucl. Phys. B617, 441 (2001); E. Pallante and A. Pich, Phys. Rev. Lett. 84, 2568 (2000), Nucl. Phys. B592, $294(2000)$.

[46] A. Alavi-Harari et al. (KTev collab.), Phys. Rev. Lett 83, 22 (1999); A. Lai et al. (NA48 collab.), Eur. Phys. J. C 22, 231 (2001).

[47] T. Ibrahim and P. Nath, Phys. Rev. D57, 478 (1998), Phys. Rev. D58, 111301 (1998).

[48] G. F. Giudice and A. Masiero, Phys. Lett. B206, 480 (1988).

[49] For alternative solutions to the $\mu$-problem based on gauged $B-L$ symmetry, see: $\mathrm{S}$. King and Q. Shafi, Phys.Lett. B422, 135 (1998); S. Jeannerot, S. Khalil, G. Lazarides and Q. Shafi, JHEP 0010, 012 (2000); K. S. Babu, B. Dutta and R. N. Mohapatra, Phys. Rev. D 65, 016005 (2002); R. Kitano and N. Okada, Prog. Theor. Phys. 106, 1239 (2001); L. J. Hall, Y. Nomura and A. Pierce, Phys. Lett. B 538, 359 (2002).

[50] See e.g. J. Ellis, J. F. Gunion, H. E. Haber, L. Roszkowski and F. Zwirner, Phys. Rev. D39, 844 (1989); L. Durand and J. L. Lopez, Phys. Lett. B217, 463 (1989).

[51] P. Harris et al. Phys. Rev. Lett. 82, 94 (1999).

[52] E. D. Commins et al, Phys. Rev. A50, 2960 (1994).

[53] For a sample see: C. H. Albright, K. S. Babu and S. M. Barr, Phys. Rev. Lett. 81, 1167 (1998) J. Sato and T. Yanagida, Phys. Lett. B 430, 127 (1998) N. Irges, S. Lavignac and P. Ramond, Phys. Rev. D 58, 035003 (1998); C. H. Albright and S. M. Barr, Phys. Lett. 
B 461, 218 (1999); G. Altarelli and F. Feruglio, Phys. Lett. B 451, 388 (1999); T. Blazek, S. Raby and K. Tobe, Phys. Rev. D62, 055001 (2000), hep-ph/9912482; K. Hagiwara and N. Okamura, Nucl. Phys. B 548, 60 (1999); M. C. Chen and K. T. Mahanthappa, Int. J. Mod. Phys. A 18, 5819 (2003); C. S. Huang, T. j. Li and W. Liao, Nucl. Phys. B 673, 331 (2003); N. Maekawa, arXiv:hep-ph/0402224. 